\title{
Analysis and optimal control of a deterministic Zika virus model
}

\author{
Dawit Denu' ${ }^{a, *}$, Hyunjin Son ${ }^{b}$ \\ a Department of Mathematics, Georgia Southern University, 11935 Abercorn Street, Savannah, GA 31419, USA. \\ ${ }^{b}$ Mathematics, Division of Science, Southern Wesleyan University, 907 Wesleyan Drive, Central, SC 29630, USA.
}

\begin{abstract}
In this paper, we consider a deterministic model explaining how Zika virus is transmitted between human and mosquito. The human population is divided into three groups as susceptible $\left(x_{1}\right)$, infected $\left(x_{2}\right)$, and treated $\left(x_{3}\right)$. Similarly, the mosquito population is divided into susceptible $\left(y_{1}\right)$ and infected $\left(y_{2}\right)$ groups. First, we conduct the local and global stability of the disease-free and endemic equilibrium points in relation to the basic reproductive number. We also study the sensitivity of the basic reproductive number and the endemic equilibrium point with respect to each parameters used in the model. Furthermore, we apply optimal control theory to show that there are cost effective control methods with the prevention effort $\left(u_{1}\right)$ of the contact between human and vector and the effort of treatment $\left(u_{2}\right)$ for human. Finally, we provide numerical simulations to support and illustrate some of the theoretical results.
\end{abstract}

Keywords: Deterministic Zika virus model, basic reproductive number, local and global stability, sensitivity analysis, optimal control.

2020 MSC: 92D30, 49Q12.

(C)2022 All rights reserved.

\section{Introduction}

The Zika virus is a generally mild illness caused when an infected mosquito of genus Aedes bites a healthy human being. The genus Aedes includes Aedes apicoargenteus, Aedes aegypti, Aedes furcifer, Aedes vitattus, Aedes luteocephalus, Aedes africanus, and Aedes hensilli. The virus is similar to those that cause dengue, hepatitis C, West Nile virus, Saint Louis encephalitis, yellow fever, hog cholera, and chikungunya. Even though the Zika virus is mainly transmitted through the bite of an infected mosquito, it can also be spread to people in other ways such as from mother to a fetus during pregnancy or during the time of birth, through infected blood and sexual contact, and others. Most people infected with the Zika virus have no signs and symptoms, while others report mild fever, rash, and joint or muscle pain. Other signs and symptoms may include headache, red eyes or conjunctivitis, and a general feeling of discomfort. Zika virus infections during pregnancy have been linked to miscarriage and microcephaly which includes birth defects such as severe microcephaly with a partly collapsed skull, brain damage, and reduced brain tissue, eye damage, joint problems including limited motion, reduced body movement due to too much

\footnotetext{
*Corresponding author

Email addresses: ddenu@georgiasouthern.edu (Dawit Denu), hson@swu .edu (Hyunjin Son)
}

doi: $10.22436 /$ jnsa.015.02.02

Received: 2021-04-27 Revised: 2021-05-17 Accepted: 2021-07-05 
muscle tone after birth and others [13]. It is also known that the Zika virus may cause other neurological disorders collectively known as Guillain-Barre syndrome (GBS). In this case, the immune system of the patient will attack his/her nerves which results in weakness and tingling in the extremities such as feet, fingers, and legs [13, 46, 47].

Since the symptoms of Zika virus infections are similar to other diseases like dengue and chikungunya, it is difficult to diagnose clinically $[29,34]$. There is no specific treatment or antiviral drug for Zika infection. Also, because there is no vaccine for the Zika virus, we consider prevention measures that are the same as for all vector-borne diseases caused by mosquitoes such as Aedes aegypti. There is much literature on prevention, especially on Zika virus and dengue fever prevention and control $[28,41,50,51]$ and mosquito bite prevention [5, 13, 45, 49].

The Zika virus was first discovered by a team of researchers in 1947 while studying yellow fever near the East African Virus Research Institute in Entebbe, Uganda. Since its first discovery, there are several major outbreaks in different parts of the world. However, according to the world health organization, the first major outbreak of Zika virus infection was reported from the Island of Yap in 2007. Similarly, there were other major outbreaks of Zika virus infections. For example, in Polynesia, Easter Island, the Cook Islands, and New Caledonia (during 2013); in Central and South America, and the Caribbean (during 2015). According to Jorg Heukelbach et al., between August and October 2015, there was an estimate of 29 cases of microcephaly in the Northeastern part of Brazil [21, 27, 30, 49, 55].

Several authors have used mathematical models to understand how fast an infectious disease can be spread and how long the disease can exist after emergence or reemergence. Thus, with the help of a mathematical model, researchers could come up with the best strategies to stop the spread of the disease, choose a better effective immunization program, allocate scarce resources to control or prevent infections and also predict the future course of an outbreak.

The biology and epidemiology of the Zika virus have been studied by several authors. Adam et al. investigated the 2013-2014 Zika virus outbreak on the six major archipelagos of French Polynesia. They analyzed the possibilities of a reemergence of the infection and also, they studied the similarity of Zika virus dynamics to other vector-borne infections such as the dengue virus in the pacific region [37]. Similar results can also be found in $[6,12,22,46]$. A Zika virus model is developed and studied by Deborah et al., for the 2015 - 2016 outbreak that happened in Colombia, El Salvador, and Suriname. They estimated the parameter distributions of the Zika virus model and also provided uncertainty quantification using Approximate Bayesian Computation [53]. In [44], Moreno et al. studied the dynamics of the Zika virus about short-term mobility between two populations. They also investigated the asymptomatic and symptomatic infected populations and estimated the basic reproductive number for a two-patch model by assuming that vectors are not moving across patches. Similar studies of the Zika virus in different countries in South America can be found here $[9,58]$. Anuwat et al. reviewed the different methods used in modeling Zika virus transmission [60]. In their review, they summarized the five basic mathematical models (compartmental, spatial, meta-population, network, and individual-based) that are used by different authors to study the transmission of vector-borne infections in general and the Zika virus in particular.

We will formulate an optimal control on the given model to derive optimal prevention of Zika virus and treatment strategies with the minimal application cost. Some literature have used control theory on HIV disease [1, 16, 31, 36], tuberculosis [32], a vector-borne disease in general [48]. There are two methods in optimal control theory, called the direct and the indirect methods. Direct methods consist in the discretization of the optimal control problem, reducing it to a nonlinear constrained optimization problem. Indirect methods are based on the Pontryagin Maximum Principle, which in turn reduces the problem to a boundary value problem [52]. In this paper, we apply the indirect method to show that there are cost effective methods to mitigate the infection.

Optimal control theory, an extension of advanced theory of calculus of variation, is helpful to derive control policies in problems related to epidemiology, medicine, economics, military science and other areas $[15,39]$. 
An optimal control problem consists of a cost functional $J(x(t), u(t))$, state variable $x(t)$, and control variable $u(t)$ where $t_{0} \leqslant t \leqslant t_{1}$. The goal is to find piece-wise continuous functions for control and state variables maximizing or minimizing a cost functional [52, 61].

The organization of the paper is as follows. In Section 2, we formulate the deterministic Zika virus model. We use the susceptible-infected-recovered (SIR) type of structure for the human population and a susceptible-infected (SI) structure for the mosquitoes. In Section 3, we analyze the model. In particular, we show that the local and global stability of the disease-free and endemic equilibrium points are completely determined by the basic reproductive number $\mathcal{R}_{0}$. We also discuss the sensitivity analysis of the basic reproductive number and endemic equilibrium point, which are the most important values in epidemiology. In Section 4, we analyze an optimal control model for the Zika virus to derive optimal prevention effort of the contact between the human and the mosquitoes and treatment strategies with minimal cost. Finally, in the last section, we provide numerical examples and simulations to support some of the theoretical results in the previous sections.

\section{Mathematical model formulation}

A compartmental framework is used to model the transmission dynamics of Zika virus. The human population is divided into three groups. Let $x_{1}(t), x_{2}(t)$, and $x_{3}(t)$ be the number of susceptible, infected, and recovered people at time $t \geqslant 0$, respectively. Similarly, the mosquito population is divided into two groups, and let $y_{1}(t)$ and $y_{2}(t)$ be the number of susceptible and infected mosquitoes at time $t \geqslant 0$, respectively.

In the study of vector-host epidemic model, it is customary to use the susceptible, exposed, infected and recovered (SEIR) compartmental type of structure for the host population, and the susceptible, exposed and infected (SEI) type for the vector population. As a result, the mathematical model will have a system of at least 7-dimensional nonlinear differential equations. In this paper, to reduce the level of complexity that arises from analyzing the system, we assume that all individuals who are exposed or already infected and are contagious are grouped under the infected group $x_{2}(t)$. Similarly, by assuming that the life span of mosquitoes is short enough compared to the host, we ignore the exposed group. Thus we consider an SIR epidemic model for human and an SI model for the mosquito population. Similar literature where an SIR type of structure for the host and an SI type for the vector can be found [8, 35, 42, 60]. The Zika virus model studied in this paper can be modified to an SEIR type for the host and SEI type for the vector, especially if one wants to include the intrinsic incubation period for the host and extrinsic incubation period in the vector group.

It is well known that, all epidemiological models have limitations in such a way that they do not represent the exact reality of the problem but rather a very simplified version of the real-world problem. Nonetheless, we can always draw some important conclusions about the infection and thus derive control mechanisms, which may include the decrease of susceptible people through vaccination, prevention, quarantine or treatment. In that regard, the model proposed in this paper has some limitations. One of the limitations is, it is assumed that the population in the SIR model is uniform and homogeneously mixed, but in reality, it is known that mixing depends on many factors including age, different geographic and socio-economic factors, individual human behavior and other similar factors. The other limitation is, this model is deterministic and it does not incorporate the effect of environmental fluctuations. As a result it is assumed that the output of the model is fully determined by the parametric and initial values. Another important aspect that can be considered in this model is the spacial migration of the host and the vector population. Thus by incorporating a population diffusion into this model, we can study the resulting parabolic system of partial differential equation to predict the transmission of the infection. 


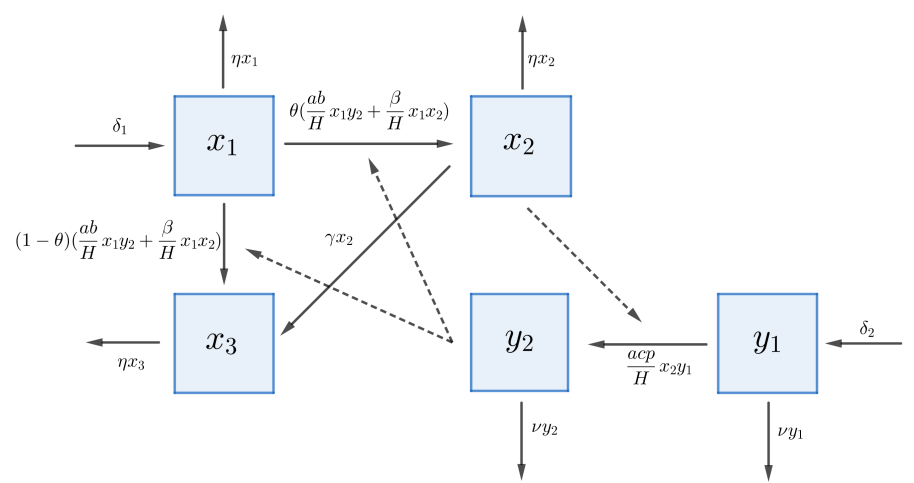

Figure 1: The flow diagram of the Zika transmission model where $x_{1}, x_{2}$, and $x_{3}$ are the number of susceptible, infected, and recovered human and $y_{1}$ and $y_{2}$ are the number of susceptible and infected mosquitoes, respectively. The solid arrows represent transitions between the different epidemiological classes, whereas the dash arrows represent interactions between human and mosquito.

Susceptible human $\left(x_{1}\right)$ can be infected if they are bitten by infectious mosquitoes $\left(y_{2}\right)$. Infectious human $\left(x_{2}\right)$ will then move to and remain in the recovered group $\left(x_{3}\right)$. Similarly, susceptible mosquitoes $\left(y_{1}\right)$ can be infected if they bite an infectious human. Based on the law of mass action, the dynamics of Zika virus transmission are described by the following 5 coupled nonlinear ordinary differential equations.

$$
\begin{aligned}
& \frac{d x_{1}(t)}{d t}=\delta_{1}-\frac{a b}{H} x_{1}(t) y_{2}(t)-\frac{\beta}{H} x_{1}(t) x_{2}(t)-\eta x_{1}(t), \\
& \frac{d x_{2}(t)}{d t}=\theta\left(\frac{a b}{H} x_{1}(t) y_{2}(t)+\frac{\beta}{H} x_{1}(t) x_{2}(t)\right)-\gamma x_{2}(t)-\eta x_{2}(t), \\
& \frac{d x_{3}(t)}{d t}=(1-\theta)\left(\frac{a b}{H} x_{1}(t) y_{2}(t)+\frac{\beta}{H} x_{1}(t) x_{2}(t)\right)+\gamma x_{2}(t)-\eta x_{3}(t), \\
& \frac{d y_{1}(t)}{d t}=\delta_{2}-\frac{a c p}{H} x_{2}(t) y_{1}(t)-v y_{1}(t), \\
& \frac{d y_{2}(t)}{d t}=\frac{a c p}{H} x_{2}(t) y_{1}(t)-v y_{2}(t),
\end{aligned}
$$

where $H(t)=x_{1}(t)+x_{2}(t)+x_{3}(t)$ and $V(t)=y_{1}(t)+y_{2}(t)$ represent the total human and mosquito population. A detailed description of the parameters used in the model with their values are given in Table 1.

Table 1: Description of parameters used in the Zika model and their range.

\begin{tabular}{|c|l|c|c|}
\hline Parameters & Description of the parameters (unit) & Range & Reference \\
\hline $\mathrm{a}$ & Mosquito biting rate (number of bites per mosquito per day) & $0.3-1$ & {$[4,27]$} \\
\hline $\mathrm{b}$ & $\begin{array}{l}\text { Transmission probability from an infected mosquito to a susceptible } \\
\text { human per bite (dimensionless) }\end{array}$ & $0.1-0.75$ & {$[4,27]$} \\
\hline $\mathrm{c}$ & $\begin{array}{l}\text { Transmission probability from an infected human to a susceptible } \\
\text { mosquito per bite (dimensionless) }\end{array}$ & $0.3-0.75$ & {$[4,27]$} \\
\hline$\beta$ & Transmission rate from infected human to susceptible human (per day) & $0.001-0.4$ & {$[27]$} \\
\hline$\theta$ & Proportion of symptomatic infections (dimensionless) & $0.1-0.8$ & {$[27]$} \\
\hline$\eta$ & Death rate of the human population (per day) & {$[2-5] \cdot 10^{-5}$} & Assumed \\
\hline$\gamma$ & Recovery rate of infected human (per day) & $0.01-0.33$ & {$[4]$} \\
\hline$p$ & $\begin{array}{l}\text { Relative human-to-mosquito transmission probability of infected hu- } \\
\text { man to susceptible mosquito per bite (dimensionless) }\end{array}$ & $0-0.5$ & {$[27]$} \\
\hline$v$ & Death rates of the susceptible and infected mosquito (per day) & $0.04-0.09$ & {$[53]$} \\
\hline$\delta_{1}$ & Recruitment rates of susceptible human (per day) & $>1$ & Assumed \\
\hline$\delta_{2}$ & Recruitment rates of susceptible mosquito (per day) & $>1$ & Assumed \\
\hline
\end{tabular}


Since $x_{3}(t)$ appears only in third equation in (2.1), we can remove it from the system and study the following equivalent 4-dimensional system of nonlinear differential equation.

$$
\begin{aligned}
& \frac{d x_{1}(t)}{d t}=\delta_{1}-\frac{a b}{H} x_{1}(t) y_{2}(t)-\frac{\beta}{H} x_{1}(t) x_{2}(t)-\eta x_{1}(t) \\
& \frac{d x_{2}(t)}{d t}=\theta\left(\frac{a b}{H} x_{1}(t) y_{2}(t)+\frac{\beta}{H} x_{1}(t) x_{2}(t)\right)-\gamma x_{2}(t)-\eta x_{2}(t) \\
& \frac{d y_{1}(t)}{d t}=\delta_{2}-\frac{a c p}{H} x_{2}(t) y_{1}(t)-v y_{1}(t) \\
& \frac{d y_{2}(t)}{d t}=\frac{a c p}{H} x_{2}(t) y_{1}(t)-v y_{2}(t)
\end{aligned}
$$

Define the set $\Omega:=\left\{\left(x_{1}, x_{2}, y_{1}, y_{2}\right) \in \mathbb{R}_{+}^{4}: x_{1}+x_{2} \leqslant \frac{\delta_{1}}{\eta}, y_{1}+y_{2}=\frac{\delta_{2}}{v}\right\}$. Adding the first 3 equations in system (2.1), we obtain

$$
\frac{d H(t)}{d t}=\delta_{1}-\eta H(t)
$$

and similarly, adding the last 2 equations we have

$$
\frac{d V(t)}{d t}=\delta_{2}-v V(t)
$$

This yields $H(t)=\frac{\delta_{1}}{\eta}+c_{1} e^{-\eta t}$ and $V(t)=\frac{\delta_{2}}{v}+c_{2} e^{-v t}$, where $c_{1}$ and $c_{2}$ are arbitrary constant. Note that $\lim _{t \rightarrow \infty} H(t)=\frac{\delta_{1}}{\eta}$ and $\lim _{t \rightarrow \infty} V(t)=\frac{\delta_{2}}{\eta}$. Thus, without loss of generality, we can assume that the limiting population of the human and mosquitoes is constant. That is, $H(t)=\frac{\delta_{1}}{\eta}$ and $V(t)=\frac{\delta_{2}}{v}$ for any $\mathrm{t} \geqslant 0$ provided that $\mathrm{H}(0)=\frac{\delta_{1}}{\eta}, \mathrm{V}(0)=\frac{\delta_{2}}{v}$ and as a result we conclude that $\Omega$ is positively invariant and globally attractive with respect to system (2.2).

\section{Analysis of the model}

\subsection{Equilibrium points and basic reproductive number}

Solving the system

$$
\frac{d x_{i}}{d t}=0, \frac{d y_{j}}{d t}=0 \quad \text { for } i, j=1,2
$$

yields two equilibrium points. These are, the disease-free equilibrium point

$$
\mathrm{E}_{0}=\left(\frac{\delta_{1}}{\eta}, 0, \frac{\delta_{2}}{v}, 0\right)
$$

and the endemic equilibrium point $E_{1}=\left(x_{1}^{*}, x_{2}^{*}, y_{1}^{*}, y_{2}^{*}\right)$, where

$$
x_{1}^{*}=\frac{\delta_{1}}{\eta}-\frac{(\gamma+\eta)}{\eta \theta} x_{2}^{*}, \quad y_{1}^{*}=\frac{\delta_{2} H}{a c p x_{2}^{*}+v H^{\prime}}, \quad y_{2}^{*}=\frac{a c p \delta_{2} x_{2}^{*}}{v\left(a c p x_{2}^{*}+v H\right)}
$$

and $x_{2}^{*}$ is the positive solution of the equation

$$
\frac{2 \eta \theta \delta_{1}-(\gamma+\eta) \eta x_{2}^{*}}{\theta \delta_{1}-(\gamma+\eta) x_{2}^{*}}=\frac{a^{2} b c p \delta_{2} x_{2}^{*}}{H v\left(a c p x_{2}^{*}+v H\right)}-\frac{\beta}{H} x_{2}^{*} .
$$

The basic reproductive number $\mathcal{R}_{0}$ is an epidemiological quantity, that represents the expected number of secondary Zika infections by a single infectious individual over the duration of infectious period with in a fully susceptible population $[2,19,20]$. We use the next generation method to obtain $\mathcal{R}_{0}$. For that 
purpose, let

$$
F\left(E_{0}\right)=\left[\begin{array}{cc}
\frac{\theta \beta \delta_{1}}{H \eta} & \frac{\theta a b \delta_{1}}{H \eta} \\
\frac{a c p \delta_{2}}{H v} & 0
\end{array}\right] \text { and } V\left(E_{0}\right)=\left[\begin{array}{cc}
\gamma+\eta & 0 \\
0 & v
\end{array}\right] .
$$

Then the next generation matrix $G\left(E_{0}\right)$ is defined by

$$
G\left(E_{0}\right)=F\left(E_{0}\right) V^{-1}\left(E_{0}\right)=\left[\begin{array}{cc}
\frac{\theta \beta \delta_{1}}{H \eta(\gamma+\eta)} & \frac{\theta a b \delta_{1}}{H \eta v} \\
\frac{a c p \delta_{2}}{H v(\gamma+\eta)} & 0
\end{array}\right]
$$

Hence $\mathcal{R}_{0}$, which is the dominant eigenvalue of $\mathrm{G}$, is given by

$$
\mathcal{R}_{0}=\frac{1}{2}\left(\frac{\theta \beta}{\gamma+\eta}+\sqrt{\frac{\theta^{2} \beta^{2}}{(\gamma+\eta)^{2}}+\frac{4 \theta \mathrm{a}^{2} \mathrm{bcp} \delta_{2}}{\mathrm{H} v^{2}(\gamma+\eta)}}\right) .
$$

\subsection{Local and global stability of the equilibrium point}

In this subsection, we discuss the local and global stability of the disease-free and endemic equilibrium points.

Theorem 3.1. The disease-free equilibrium point $\mathrm{E}_{0}$ is locally asymptotically stable in $\Omega$ if $\mathcal{R}_{0}<1$.

Proof. To prove the local stability of $\mathrm{E}_{0}$, we need to show that all the real roots of the eigenvalues of the Jacobian matrix $J\left(E_{0}\right)$ are non-positive. To that end, we have

$$
J\left(E_{0}\right)=\left[\begin{array}{cccc}
-\eta & \frac{\beta \delta_{1}}{H \eta} & 0 & -\frac{a b \delta_{1}}{H \eta} \\
0 & \frac{\theta \beta \delta_{1}}{H \eta}-\gamma-\eta & 0 & \frac{\theta a b \delta_{1}}{H \eta} \\
0 & -\frac{a c p \delta_{2}}{H v} & -v & 0 \\
0 & \frac{a c p \delta_{2}}{H v} & 0 & -v
\end{array}\right] .
$$

The eigenvalues of $J\left(E_{0}\right)$ satisfy

$$
(\lambda+\eta)(\lambda+v)\left(\lambda^{2}+k_{0} \lambda+k_{1}\right)=0
$$

where

$$
\mathrm{k}_{0}:=-\frac{\theta \beta \delta_{1}}{\mathrm{H} \eta}+\gamma+\eta+v
$$

and

$$
\mathrm{k}_{1}:=v\left(\gamma+\eta-\frac{\theta \beta \delta_{1}}{\mathrm{H} \eta}\right)-\frac{\mathrm{a}^{2} \operatorname{cp} \theta \delta_{1} \delta_{2}}{\mathrm{H}^{2} v \eta} .
$$

Now if $\mathcal{R}_{0}<1$, we have

$$
\frac{\theta \beta \delta_{1}}{H \eta(\gamma+\eta+\nu)} \leqslant \frac{\theta \beta \delta_{1}}{H \eta(\gamma+\eta)} \leqslant R_{0}<1 .
$$

Thus $k_{0}>0$. Also if $\mathcal{R}_{0}<1$, then

$$
\frac{\theta \beta \delta_{1}}{\mathrm{H} \eta}+\frac{\theta \mathrm{a}^{2} \mathrm{bcp} \delta_{1} \delta_{2}}{\mathrm{H}^{2} v^{2} \eta}-\gamma-\eta<0 .
$$

Note that

$$
k_{1}=\left(\gamma+\eta-\frac{\theta \beta \delta_{1}}{H \eta}-\frac{\theta a^{2} b c p \delta_{1} \delta_{2}}{H^{2} v^{2} \eta}\right) v>0 .
$$

In conclusion, all the eigenvalues of the Jacobian matrix have a negative real part and thus the disease-free equilibrium point $E_{0}$ is locally stable.

To prove the global stability of $E_{0}$, we use the Lasalle's invariance principle stated below $[38,57]$. 
Theorem 3.2. Let $\Omega \subset \mathrm{D}$ be a compact set that is positively invariant with respect to $x^{\prime}=\mathrm{f}(\mathrm{x})$. Let $\mathrm{V}: \mathrm{D} \rightarrow \mathbb{R}$ be a $\mathrm{C}^{1}$-function such that $\mathrm{V}^{\prime}(\mathrm{t}) \leqslant 0$ on $\Omega$. Let $\mathrm{E}=\left\{x \in \Omega: \mathrm{V}^{\prime}(\mathrm{t})=0\right\}$ and $\mathrm{M}$ be the largest invariant set in $\mathrm{E}$. Then every solution starting in $\Omega$ approaches $M$ as $\mathrm{t} \rightarrow \infty$.

Theorem 3.3. If $\mathcal{R}_{0}<1$, then the disease-free equilibrium point is globally asymptotically stable.

Proof. Define a function

$$
\mathrm{V}\left(\mathrm{x}_{1}, \mathrm{x}_{2}, \mathrm{y}_{1}, \mathrm{y}_{2}\right)=\mathrm{x}_{2}+\frac{\theta \mathrm{ab} \delta_{1}}{v \mathrm{H} \eta} \mathrm{y}_{2}
$$

Clearly, $V\left(x_{1}, x_{2}, y_{1}, y_{2}\right) \geqslant 0$ along the solution of system (2.2). Using the fact that $y_{1}=\frac{\delta_{2}}{v}-y_{2}$, we have the following result.

$$
\begin{aligned}
\frac{d V}{d t} & =\frac{d x_{2}}{d t}+\frac{\theta a b \delta_{1}}{v H \eta} \frac{d y_{2}}{d t} \\
& =\theta\left(\frac{a b}{H} x_{1} y_{2}+\frac{\beta}{H} x_{1} x_{2}\right)-\gamma x_{2}-\eta x_{2}+\frac{\theta a b \delta_{1}}{v H \eta}\left(\frac{a c p \delta_{2}}{H v} x_{2}-\frac{a c p}{H} x_{2} y_{2}-v y_{2}\right) \\
& \leqslant x_{2}\left(\frac{\theta \beta \delta_{1}}{H \eta}-\gamma-\eta+\frac{\theta a^{2} b c p \delta_{1} \delta_{2}}{H^{2} v^{2} \eta}-\frac{\theta a^{2} b c p \delta_{1}}{H^{2} v \eta} y_{2}\right) \\
& =x_{2}\left((\gamma+\eta)\left[\frac{\theta \beta \delta_{1}}{H \eta(\gamma+\eta)}+\frac{\theta a^{2} b c p \delta_{1} \delta_{2}}{H^{2} v^{2} \eta(\gamma+\eta)}-1\right]-\frac{\theta a^{2} b c p \delta_{1}}{H^{2} v \eta} y_{2}\right) .
\end{aligned}
$$

Notice that if $\mathcal{R}_{0}<1$, it follows that $\frac{\theta \beta \delta_{1}}{\mathrm{H \eta}(\gamma+\eta)}+\frac{\theta \mathrm{a}^{2} \mathrm{bcp} \delta_{1} \delta_{2}}{\mathrm{H}^{2} \gamma^{2} \eta(\gamma+\eta)}<1$ and then $\frac{\mathrm{d} V}{\mathrm{dt}} \leqslant 0$. Thus by Theorem 3.2, we conclude that the disease-free equilibrium point $\mathrm{E}_{0}$ is globally asymptotically stable.

Theorem 3.4. The endemic equilibrium point $\mathrm{E}_{1}$ is locally asymptotically stable if $\mathcal{R}_{0}>1$.

Proof. The proof is similar to Theorem 3.1. The characteristic equation of the Jacobian matrix evaluated at $E_{1}$ is given by

$$
\begin{gathered}
\lambda^{3}-\left(a_{1}+a_{2}+a_{8}\right) \lambda^{2}+\left(a_{1} a_{5}+a_{1} a_{8}+a_{5} a_{8}-a_{2} a_{4}+a_{6} a_{7}\right) \lambda \\
+\left(-a_{1} a_{6} a_{7}+a_{2} a_{4} a_{8}-a_{3} a_{4} a_{7}-a_{1} a_{5} a_{8}\right)=0,
\end{gathered}
$$

where

$$
\begin{array}{llrl}
a_{1}=-\frac{a b y_{2}^{*}}{H}-\frac{\beta x_{2}^{*}}{H}-\eta<0, & a_{2}=-\frac{\beta x_{1}^{*}}{H}<0, & a_{3}=-\frac{a b x_{1}^{*}}{H}<0, \\
a_{4}=\frac{\theta a b y_{2}^{*}}{H}+\frac{\theta \beta x_{2}^{*}}{H}>0, & a_{5}=\frac{\theta \beta x_{1}^{*}}{H}-\gamma-\eta<0, & a_{6}=\frac{\theta a b x_{1}^{*}}{H}>0, \\
a_{7}=\frac{a c p \delta_{2}}{H v}-\frac{a c p y_{2}^{*}}{H}>0, & a_{8}=-\frac{a c p x_{2}^{*}}{H}-v<0 . &
\end{array}
$$

Now if we let

$$
u=-\left(a_{1}+a_{2}+a_{8}\right), v=a_{1} a_{5}+a_{1} a_{8}+a_{5} a_{8}-a_{2} a_{4}+a_{6} a_{7}, z=-a_{1} a_{6} a_{7}+a_{2} a_{4} a_{8}-a_{3} a_{4} a_{7}-a_{1} a_{5} a_{8},
$$

then $u>0, v>0$, and $z>0$. Also we have

$$
\begin{aligned}
u v-z= & a_{1} a_{2} a_{4}-a_{1}^{2} a_{5}+a_{2} a_{4} a_{5}-a_{1} a_{5}^{2}-a_{3} a_{4} a_{7}+2 a_{1} a_{6} a_{7}+a_{5} a_{6} a_{7} \\
& +a_{1}^{2} a_{8}-2 a_{1} a_{5} a_{8}-a_{5}^{2} a_{8}-a_{6} a_{7} a_{8}-a_{1} a_{8}^{2}-a_{5} a_{8}^{2}>0 .
\end{aligned}
$$

Thus according to the Hurwitz criterion, the endemic equilibrium $E_{1}$ is locally asymptotically stable [17]. 
Next, we use the Poincaré-Bendixon theorem to prove the global stability of the endemic equilibrium point $\mathrm{E}_{1}[11,40,54]$.

Theorem 3.5. Let $\mathrm{x} \rightarrow \mathrm{f}(\mathrm{x}) \in \mathbb{R}^{\mathrm{n}}$ be a $\mathrm{C}^{1}$ function for $\mathrm{x} \in \mathrm{D} \subset \mathbb{R}^{\mathrm{n}}$ and consider the system of differential equations $x^{\prime}=\mathrm{f}(\mathrm{x})$. Assume that

1. there exists a compact absorbing set $\mathrm{K} \subset \mathrm{D}$ and the above system has a unique equilibrium $\overline{\mathrm{x}} \in \mathrm{D}$;

2. $\bar{x}$ is locally asymptotically stable;

3. the system satisfies the Poincaré-Bendixon property;

4. any periodic orbit of the system is asymptotically orbitally stable.

Then the unique equilibrium $\bar{x}$ is globally asymptotically stable in D.

Note that $\Omega$ is bounded. Thus, in order to show that system (2.2) has a compact absorbing set, it is sufficient to prove system (2.2) is uniformly persistent $[40,59]$. That is, we have to show that there exists $k>0$ such that, the solution of system (2.2) satisfies the condition

$$
\liminf _{t \rightarrow \infty}\left|\left(x_{1}(t), x_{2}(t), y_{1}(t), y_{2}(t)\right)\right| \geqslant k
$$

for any initial value $x_{1}(0) \geqslant 0, x_{2}(0) \geqslant 0, y_{1}(0) \geqslant 0, y_{2}(0) \geqslant 0$.

Lemma 3.6. System (2.2) is uniformly persistent if $\mathcal{R}_{0}>1$.

Proof. By contradiction, suppose there exists a solution $x_{1}(t), x_{2}(t), y_{1}(t)$, and $y_{2}(t)$ of system (2.2), such that $x_{1}(0)>0, x_{2}(0)>0, y_{1}(0)>0, y_{2}(0)>0$ and

$$
\lim _{t \rightarrow \infty} x_{1}(t)=\frac{\delta_{1}}{\eta}, \lim _{t \rightarrow \infty} x_{2}(t)=0, \lim _{t \rightarrow \infty} y_{1}(t)=\frac{\delta_{2}}{v}, \lim _{t \rightarrow \infty} y_{2}(t)=0 .
$$

If $\mathcal{R}_{0}>1$, then $\frac{\theta \beta}{\gamma+\eta}+\frac{\theta \mathrm{a}^{2} b c p \delta_{2}}{\mathrm{H} v^{2}(\gamma+\eta)}>1$ and thus, there exists $\epsilon>0$ such that

$$
\theta \beta\left(\frac{\delta_{1}}{\eta}-\epsilon\right) v+\theta a^{2} b c p\left(\frac{\delta_{2}}{v}-\epsilon\right)-H v(\gamma+\eta)>0 .
$$

Equation (3.3) implies that for $\epsilon>0$, there is $t_{0}>0$ such that for any $t>t_{0}$ we have

$$
\frac{\delta_{1}}{\eta}-\epsilon<x_{1}(t)<\frac{\delta_{1}}{\eta}+\epsilon, x_{2}(t)<\epsilon, \frac{\delta_{2}}{v}-\epsilon<y_{1}(t)<\frac{\delta_{2}}{v}+\epsilon, y_{2}(t)<\epsilon .
$$

Thus if $t \geqslant t_{0}$ we have

$$
\frac{\mathrm{d} x_{2}}{\mathrm{dt}}=\theta\left(\frac{\mathrm{ab}}{\mathrm{H}} x_{1} \mathrm{y}_{2}+\frac{\beta}{\mathrm{H}} x_{1} x_{2}\right)-\gamma x_{2}-\eta x_{2} \geqslant\left(\frac{\theta \beta}{\mathrm{H}}\left(\frac{\delta_{1}}{\eta}-\epsilon\right)-\gamma-\eta\right) x_{2}+\theta \frac{\mathrm{ab}}{\mathrm{H}}\left(\frac{\delta_{1}}{\eta}-\epsilon\right) \mathrm{y}_{2},
$$

and

$$
\frac{d y_{2}}{d t}=\frac{a c p}{H} x_{2} y_{1}-v y_{2} \geqslant \frac{a c p}{H}\left(\frac{\delta_{2}}{v}-\epsilon\right) x_{2}-v y_{2} .
$$

Now consider the following system of linear differential equations:

$$
\left\{\begin{array}{l}
\frac{d x}{d t}=\left(\frac{\theta \beta}{H}\left(\frac{\delta_{1}}{\eta}-\epsilon\right)-\gamma-\eta\right) x+\theta \frac{a b}{H}\left(\frac{\delta_{1}}{\eta}-\epsilon\right) y, \\
\frac{d y}{d t}=\frac{a c p}{H}\left(\frac{\delta_{2}}{v}-\epsilon\right) x-v y, \\
x\left(t_{0}\right)=x_{2}\left(t_{0}\right)>0, y\left(t_{0}\right)=y_{2}\left(t_{0}\right)>0 .
\end{array}\right.
$$


Note that

$$
\mathrm{Q}:=\left[\begin{array}{cc}
\left(\frac{\theta \beta}{\mathrm{H}}\left(\frac{\delta_{1}}{\eta}-\epsilon\right)-\gamma-\eta\right) & \theta \frac{\mathrm{ab}}{\mathrm{H}}\left(\frac{\delta_{1}}{\eta}-\epsilon\right) \\
\frac{\mathrm{acp}}{\mathrm{H}}\left(\frac{\delta_{2}}{v}-\epsilon\right) & -v
\end{array}\right],
$$

is a quasi-positive matrix and

$$
\operatorname{det}(\mathrm{Q})=-\frac{1}{\mathrm{H}}\left[\left(\theta \beta\left(\frac{\delta_{1}}{\eta}-\epsilon\right)-\gamma-\eta\right) \nu+\frac{\theta \mathrm{a}^{2} \mathrm{bcp}}{\mathrm{H}}\left(\frac{\delta_{1}}{\eta}-\epsilon\right)\left(\frac{\delta_{2}}{v}-\epsilon\right)\right]<0 .
$$

Thus by the Perron-Frobenius theorem there is a vector $v>0$ corresponding to the positive eigenvalue $\lambda$ of $Q$ such that $Q v=\lambda v$. Therefore, for any initial value $x\left(t_{0}\right)>0, y\left(t_{0}\right)>0$ the solution of system (3.4) is unbounded. That is $x_{2}(t) \rightarrow \infty$ and $y_{2}(t) \rightarrow \infty$ as $t \rightarrow \infty$ which is a contradiction. In conclusion, system (2.2) is uniformly persistent and thus it has a compact absorbing set.

To show that system (2.2) satisfies the Poincaré-Bendixon property, it is sufficient to prove that the system is competitive $[11,40]$. That is, for some diagonal matrix $\mathrm{D}=\operatorname{diag}\left(\zeta_{1}, \zeta_{2}, \zeta_{3}, \ldots, \zeta_{n}\right)$, DJD has nonpositive off-diagonals where $J$ is the Jacobian matrix associated with system (2.2) and $\zeta_{i} \in\{-1,1\}$ for $i=$ $1,2, \ldots, n$.

Lemma 3.7. If $\mathcal{R}_{0}>1$, then system (2.2) is competitive in $\Omega$.

Proof. Since $y_{1}(t)=\frac{\delta_{2}}{v}-y_{2}(t)$, we have $\frac{d y_{2}}{d t}=\frac{a c p}{H}\left(\frac{\delta_{2}}{v}-y_{2}\right)-v y_{2}$. Thus the Jacobian matrix of system (2.2) is given by

$$
J=\left[\begin{array}{ccc}
-\frac{a b}{H} y_{2}-\frac{\beta}{H} x_{2}-\eta & -\frac{\beta}{H} x_{1} & -\frac{a b}{H} x_{1} \\
\frac{\theta a b}{H} y_{2}+\frac{\theta \beta}{H} x_{2} & \frac{\theta \beta}{H} x_{1}-\gamma-\eta & \frac{\theta a b}{H} x_{1} \\
0 & \frac{a c p \delta_{2}}{H v}-\frac{a c p}{H} y_{2} & -\frac{a c p}{H} x_{2}-v
\end{array}\right]
$$

Let $\mathrm{D}=\operatorname{diag}(-1,1,-1)$ then,

$$
\mathrm{DJD}=\left[\begin{array}{ccc}
-\frac{\mathrm{ab}}{\mathrm{H}} y_{2}-\frac{\beta}{\mathrm{H}} x_{2}-\eta & -\frac{\beta}{\mathrm{H}} x_{1} & -\frac{\mathrm{ab}}{\mathrm{H}} x_{1} \\
-\frac{\theta a b}{\mathrm{H}} y_{2}-\frac{\theta \beta}{H} x_{2} & \frac{\theta \beta}{H} x_{1}-\gamma-\eta & -\frac{\theta a b}{H} x_{1} \\
0 & \frac{a c p \delta_{2}}{H v}-\frac{a c p}{H} y_{2} & -\frac{a c p}{H} x_{2}-v
\end{array}\right]
$$

Note that the off-diagonal elements of DJD are all non-positive. Thus system (2.2) is competitive in $\Omega$.

Next, we use the following theorem to show that any periodic orbit of system (2.2) if it exists, is asymptotically stable $[14,40]$.

Theorem 3.8. A periodic orbit $\Gamma=\{\mathrm{p}(\mathrm{t}): 0 \leqslant \mathrm{t}<\omega\}$ of the differential equation $\mathrm{x}^{\prime}=\mathrm{f}(\mathrm{x})$ is orbitally asymptotically stable with asymptotic phase if the linear system

$$
z^{\prime}(t)=\frac{\partial f^{[2]}}{\partial x}(p(t)) z(t)
$$

is asymptotically stable, where $\frac{\partial \mathrm{f}^{[2]}}{\partial \mathrm{x}}$ is the second additive compound matrix of the Jacobian matrix $\frac{\partial \mathrm{f}}{\partial \mathrm{x}}$.

Using Theorem 3.8, we prove the following result.

Theorem 3.9. Any periodic solution to the system (2.2), if it exists, is asymptotically orbitally stable.

Proof. Suppose the solution of system (2.2) is periodic with period $\tau>0$. The corresponding second additive compound matrix of the system is given by

$$
\mathrm{J}^{[2]}=\left[\begin{array}{ccc}
-\frac{\mathrm{ab}}{\mathrm{H}} y_{2}-\frac{\beta}{H} x_{2}-2 \eta+\frac{\theta \beta}{H} x_{1}-\gamma & \frac{\theta a b}{H} x_{1} & \frac{a b}{H} x_{1} \\
\frac{a c p \delta_{2}}{H}-\frac{a c p}{H} y_{2} & -\frac{a b}{H} y_{2}-\frac{\beta}{H} x_{2}-\eta-\frac{a c p}{H} x_{2}-v & -\frac{\beta}{H} x_{1} \\
0 & \frac{\theta a b}{H} y_{2}+\frac{\theta \beta}{H} x_{2} & \frac{\theta \beta}{H} x_{1}-\gamma-\eta-\frac{a c p}{H} x_{2}-v
\end{array}\right] .
$$

For any initial value $(x(0), y(0), z(0)) \in \mathbb{R}_{+}^{3}$, let $(x(t), y(t), z(t)) \in \mathbb{R}_{+}^{3}$ be the solution of system (2.2). Then 
the second compounded system is given by

$$
\begin{aligned}
& \frac{d x}{d t}=\left(-\frac{a b}{H} y_{2}-\frac{\beta}{H} x_{2}-2 \eta+\frac{\theta \beta}{H} x_{1}-\gamma\right) x+\frac{\theta a b}{H} x_{1} y+\frac{a b}{H} x_{1} z, \\
& \frac{d y}{d t}=\left(\frac{a c p \delta_{2}}{H v}-\frac{a c p}{H} y_{2}\right) x+\left(-\frac{a b}{H} y_{2}-\frac{\beta}{H} x_{2}-\eta-\frac{a c p}{H} x_{2}-v\right) y-\frac{\beta}{H} x_{1} z, \\
& \frac{d z}{d t}=\left(\frac{\theta a b}{H} y_{2}+\frac{\theta \beta}{H} x_{2}\right) y+\left(\frac{\theta \beta}{H} x_{1}-\gamma-\eta-\frac{a c p}{H} x_{2}-v\right) z .
\end{aligned}
$$

Let

$$
V\left(x, y, z, x_{2}, y_{2}\right):=\sup \left\{\theta|x|, \frac{x_{2}}{y_{2}}(|y|+|z|)\right\} .
$$

Then we have the following differential inequalities.

$$
\begin{gathered}
D_{+}|x(t)| \leqslant \theta\left(-\frac{a b}{H} y_{2}-\frac{\beta}{H} x_{2}-2 \eta+\frac{\theta \beta}{H} x_{1}-\gamma\right) x+\left(|y+z| \frac{a b x_{1} x_{2}}{H y_{2}}\right) \frac{y_{2}}{x_{2}}, \\
D_{+}\left\{\frac{x_{2}}{y_{2}}(|y(t)+z(t)|)\right\} \leqslant \frac{x_{2}}{y_{2}}\left(\frac{a c p \delta_{2}}{H v}-\frac{a c p y_{2}}{H}\right) x-\left(\frac{a c p x_{2}}{H}+\eta+v-\frac{x_{2}^{\prime}}{x_{2}}+\frac{y_{2}^{\prime}}{y_{2}}\right)(|y+z|) \frac{x_{2}}{y_{2}} .
\end{gathered}
$$

Let

$$
\begin{aligned}
& f_{1}:=-\frac{a b}{H} y_{2}-\frac{\beta}{H} x_{2}-2 \eta+\frac{\beta}{H} x_{1}-\gamma+\frac{\theta a b x_{1} y_{2}}{H x_{2}}, \\
& f_{2}:=\left(\frac{a c p \delta_{2}}{H v} \frac{x_{2}}{y_{2}}-\frac{a c p}{H} x_{2}\right)-\left(\frac{a c p x_{2}}{H}+\eta+v-\frac{x_{2}^{\prime}}{x_{2}}+\frac{y_{2}^{\prime}}{y_{2}}\right) .
\end{aligned}
$$

From system (2.2) we have the following equations:

$$
\frac{x_{2}^{\prime}}{x_{2}}=\frac{\theta a b x_{1} y_{2}}{H x_{2}}+\frac{\beta}{H} x_{1}-\gamma-\eta, \quad \frac{y_{2}^{\prime}}{y_{2}}=\frac{a c p \delta_{2} x_{2}}{H v y_{2}}-\frac{a c p x_{2}}{H}-v .
$$

Using equations (3.6) and (3.7) we obtain the following inequalities.

$$
f_{1} \leqslant \frac{x_{2}^{\prime}}{x_{2}}-\frac{a b y_{2}}{H}-\frac{\beta x_{2}}{H}-\eta \leqslant \frac{x_{2}^{\prime}}{x_{2}}-\eta, \text { and } f_{2} \leqslant \frac{x_{2}^{\prime}}{x_{2}}-\eta .
$$

Thus

$$
\sup \left\{f_{1}(t), f_{2}(t)\right\} \leqslant \frac{x_{2}^{\prime}}{x_{2}}-\eta
$$

Finally, from (3.5) and (3.6) it follows that

$$
D_{+} V(t) \leqslant \sup \left\{f_{1}, f_{2}\right\} V(t) \leqslant\left(\frac{x_{2}^{\prime}}{x_{2}}-\eta\right) V(t)
$$

Integrating inequality (3.8) on $[0, \tau]$ we have,

$$
\int_{0}^{\tau} \sup \left\{f_{1}(t), f_{2}(t)\right\} d t \leqslant \int_{0}^{\tau}\left(\frac{x_{2}^{\prime}}{x_{2}}-\eta\right) d t=\left(\ln \left(x_{2}(\tau)\right)-\ln \left(x_{2}(0)\right)\right)-\eta \tau=-\eta \tau .
$$

Hence it follows that $\mathrm{V}(\mathrm{t}) \leqslant c e^{-\eta t}$ and thus, $\mathrm{V}(\mathrm{t}) \rightarrow 0$ as $\mathrm{t} \rightarrow \infty$. This implies that $x(\mathrm{t}), \mathrm{y}(\mathrm{t}), z(\mathrm{t}) \rightarrow 0$ as $t \rightarrow \infty$. As a result, the second compound system is asymptotically stable and thus, by Theorem 3.8 the periodic orbit of system is asymptotically orbitally stable. In conclusion, the above discussion is summarized in the following theorem.

Theorem 3.10. The endemic equilibrium point $\mathrm{E}_{1}$ is globally asymptotically stable if $\mathcal{R}_{0}>1$. 
Simulation of the deterministic Zika model is presented in Figures 2 and 3 for different values of parameters listed in Table 2. For the numerical simulation, we used $\frac{1}{60}$ for $\eta$ instead of $\frac{1}{60 \times 365}$. In that way, we can see the same result in a very short period of time. Otherwise, we need to wait for a long time to see the asymptotic behavior of the solution. The set of parameter values listed under value 1 yields $\mathcal{R}_{0}=0.2415$. Thus according to Theorems 3.1 and 3.3, the solution of system (2.2) will converge to the disease-free equilibrium point $E_{0}$. Similarly, the set of parameter values under the column value 2 results $\mathcal{R}_{0}=3.81$. Therefore, by Theorems 3.4 and 3.10 the trajectories of the solution of system (2.2) will converge to the endemic equilibrium point $E_{1}$.

Table 2: Input parameter values used to simulate the trajectories of the solution of the model as shown in Figures 2 and 3 . The values under the column value 1 will result $\mathcal{R}_{0}=0.2415<1$ and the parameters under value 2 yields $\mathcal{R}_{0}=3.81>1$.

\begin{tabular}{|c|c|c|l|}
\hline & Value 1 & Value 2 & Description of the parameters (units) \\
\hline $\mathrm{a}$ & 0.4 & 0.8 & Mosquito biting rate (number of bites per mosquito per day) \\
\hline $\mathrm{b}$ & 0.4 & 0.4 & $\begin{array}{l}\text { Transmission probability from an infected mosquito to a susceptible human (dimen- } \\
\text { sionless) }\end{array}$ \\
\hline $\mathrm{c}$ & 0.3 & 0.3 & $\begin{array}{l}\text { Transmission probability from an infected human to a susceptible mosquito (dimen- } \\
\text { sionless) }\end{array}$ \\
\hline$\theta$ & 0.001 & 0.4 & Transmission rate from infected human to susceptible human (per day) \\
\hline$\eta$ & 0.1 & 0.8 & Proportion of symptomatic infections (dimensionless) \\
\hline$\gamma$ & $1 / 60$ & $1 / 60$ & Death rate of the human population (per day) \\
\hline$p$ & 0.07 & 0.07 & Recovery rate of infected human (per day) \\
\hline$v$ & 0.1 & 0.1 & $\begin{array}{l}\text { Relative human-to-mosquito transmission probability of infected human to suscepti- } \\
\text { ble mosquito (dimensionless) }\end{array}$ \\
\hline$\delta_{1}$ & 5 & $1 / 14$ & Death rates of the susceptible and infected mosquito (per day) \\
\hline$\delta_{2}$ & 40 & 2 & Recruitment rates of susceptible human (per day) [7] \\
\hline
\end{tabular}

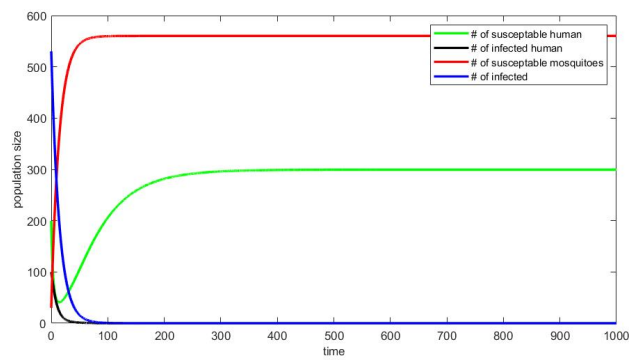

Figure 2: Trajectories of solution of the deterministic Zika model for $\mathcal{R}_{0}=0.2415<1$. The disease-free equilibrium point is calculated to be $E_{0}=(300,0,560,0)$ and the simulation also shows that the trajectories of the solution converge to $E_{0}$.

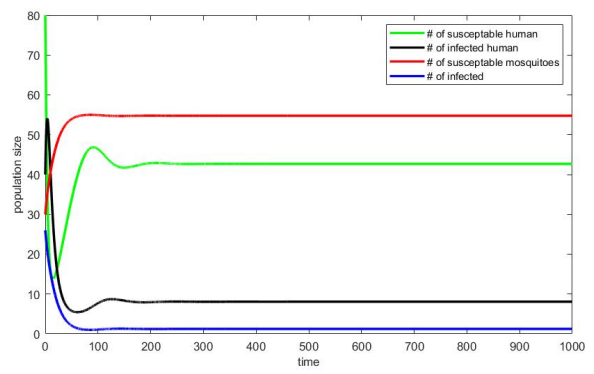

Figure 3: Trajectories of solution of the deterministic Zika model for $\mathcal{R}_{0}=3.81>1$. Using equations (3.1)-(3.2) the endemic equilibrium point is calculated to be $E_{1}=(41.602,9.471,54.855,2.112)$. The figure confirms that the trajectories of the solution converge to $E_{1}$. 
3.3. Sensitivity analysis of the basic reproductive number $\mathcal{R}_{0}$ and the endemic equilibrium point $\mathrm{E}_{1}$.

In theoretical epidemiology, the basic reproductive number and endemic equilibrium point are the two most important constants in determining whether the infection will be cleared or persist in the population. Thus, in order to fully understand which parameters are most highly correlated with the values of $\mathcal{R}_{0}$ and $E_{1}$, we have to conduct sensitivity analysis. It also helps in analyzing how sensitive $\mathcal{R}_{0}$ and $E_{1}$ are, by the changes in one input parameter while keeping the other inputs constant. For that purpose, let $\mu$ be a generic parameter representing any of the parameters used in the model. The normalized sensitivity index of $\mathcal{R}_{0}$ with respect to $\mu$ is denoted by $\mathrm{SI}_{\mu}$, and is defined as $\mathrm{SI}_{\mu}=\frac{\partial \mathcal{R}_{0}}{\partial \mu} \frac{\mu}{\mathcal{R}_{0}}$. The following values of parameters are used to calculate the sensitivity indexes for each parameters.

$$
\begin{aligned}
& a=0.8, \quad b=0.4, \quad c=0.3, \quad \beta=0.4, \quad \theta=0.8, \\
& \gamma=0.07, \quad p=0.1, \quad v=0.0714, \quad \delta_{1}=0.05, \quad \delta_{2}=4, \quad \eta=4.57 \times 10^{-5} \text {, }
\end{aligned}
$$

As it can be seen in Figure 4, the sensitivity indexes of $\gamma, \eta, v$ and $H$ are negative, while the remaining indexes $\beta, a, b, c, \theta, p, \delta_{2}$ are positive. The negative sensitivity indexes indicate that the parameter and the basic reproductive number are inversely proportional. Thus, increasing one will decrease the other value. Similarly, the positive sensitivity indexes show that there is a direct relation between the parameter and the basic reproductive number. From Figure 4 it follows that, the most sensitive parameter for $R_{0}$ is the proportion of symptomatic infection $(\theta)$, followed by the transmission rate from infected human to susceptible human $(\beta)$ and the recovery rate of infected human $(\gamma)$.

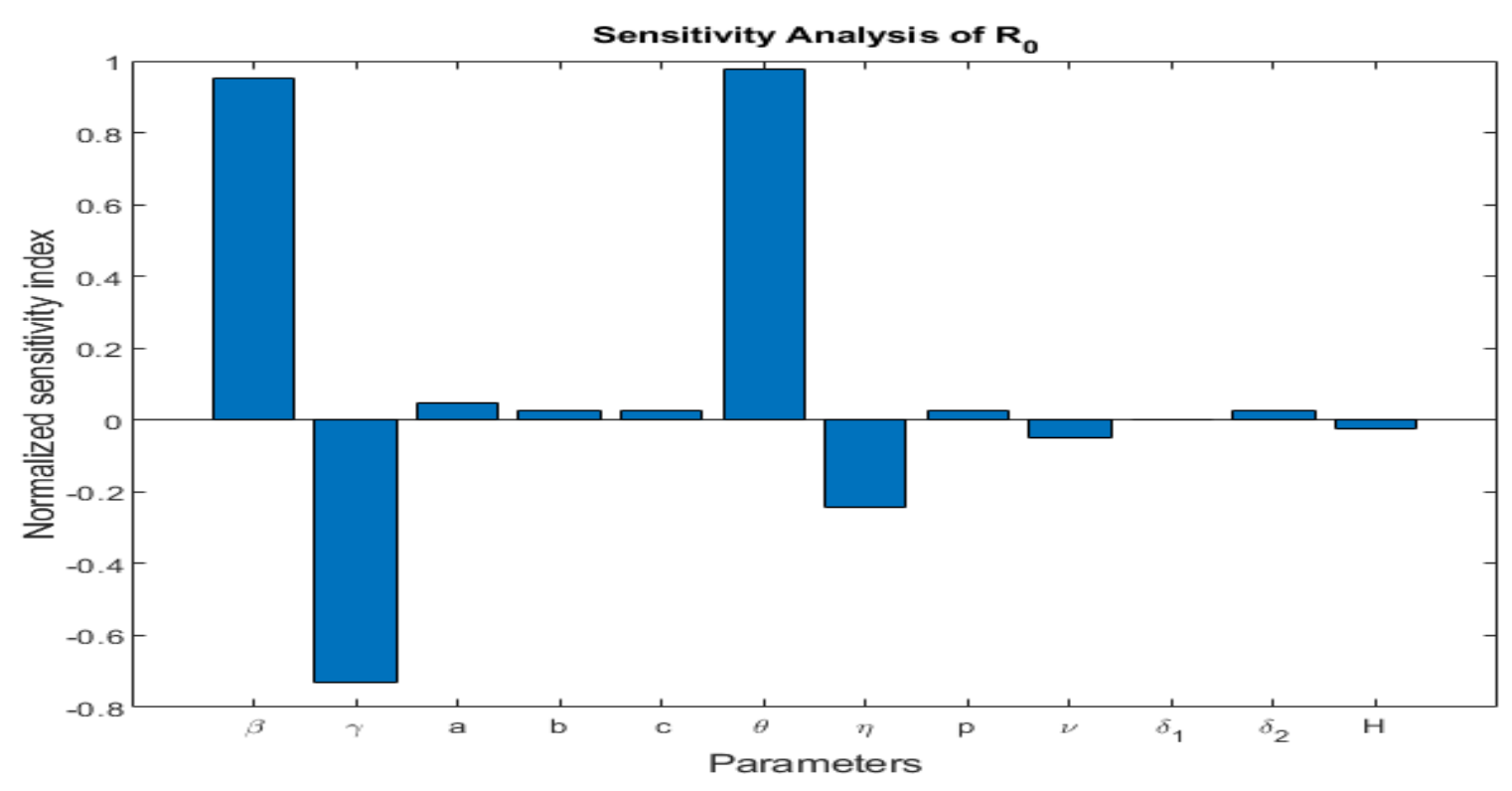

Figure 4: Sensitivity indexes of $\mathcal{R}_{0}$ with respect to the parameters used in the model.

Similarly, we discuss the sensitivity analysis of the parameters used in the model with respect to each components $x_{1}^{*}, x_{2}^{*}, y_{1}^{*}$, and $y_{2}^{*}$ of the endemic equilibrium point $E_{1}$. The same values of parameters are used to calculate the indexes. From Figure 5 we can observe that $H, \theta$ and $\beta$ are the most sensitive parameters for $x_{1}^{*}$. The most sensitive parameters for $x_{2}^{*}$ are $\delta_{1}$ and $\theta$ followed by $\gamma$. Similarly, the most sensitive parameters for $x_{3}^{*}$ are $\delta_{2}$ and $v$. Finally $v$ followed by $\theta$ and $\delta_{1}$ are the most sensitive parameters for $x_{4}^{*}$. 


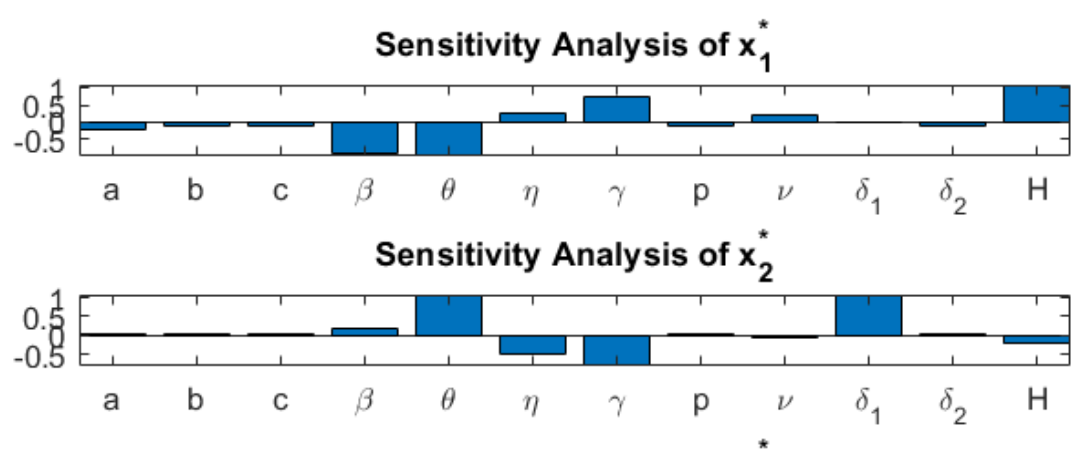

Sensitivity Analysis of $y_{1}^{*}$

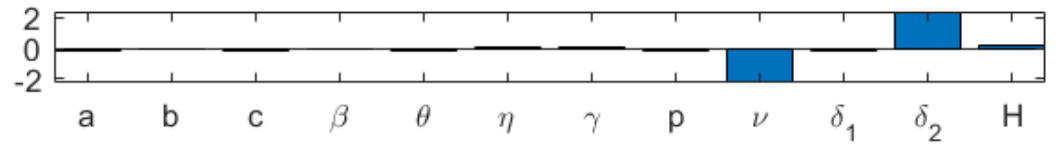

Sensitivity Analysis of $\mathrm{y}_{2}^{*}$

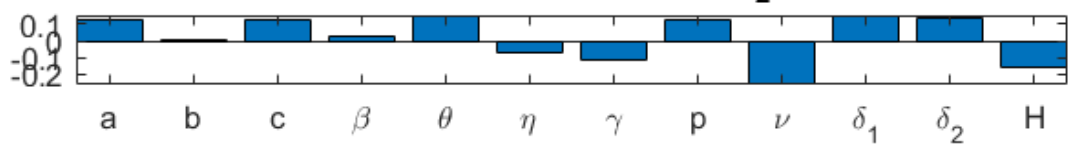

Figure 5: Sensitivity indexes of the endemic equilibrium point $E_{1}$ with respect to the parameters used in the model.

\section{Optimal control}

\subsection{A model for optimal control}

We consider two control functions $u_{1}(t)$ for the prevention effort of the contact between human and vector and $u_{2}(t)$ for the treatment for human. Thus the new system of differential equations with the control functions is given as follows.

$$
\begin{aligned}
\frac{d x_{1}}{d t} & =\delta_{1}-\frac{a b}{H}\left(1-u_{1}(t)\right) x_{1} y_{2}-\frac{\beta}{H} x_{1} x_{2}-\eta x_{1}, \\
\frac{d x_{2}}{d t} & =\theta\left(\frac{a b}{H}\left(1-u_{1}(t)\right) x_{1} y_{2}+\frac{\beta}{H} x_{1} x_{2}\right)-\gamma x_{2}-\eta x_{2}-r u_{2}(t) x_{2}, \\
\frac{d x_{3}}{d t} & =(1-\theta)\left(\frac{a b}{H}\left(1-u_{1}(t)\right) x_{1} y_{2}+\frac{\beta}{H} x_{1} x_{2}\right)+\gamma x_{2}-\eta x_{3}+r u_{2}(t) x_{2}, \\
\frac{d y_{1}}{d t} & =\delta_{2}-\frac{a c p}{H}\left(1-u_{1}(t)\right) x_{2} y_{1}-v y_{1}, \\
\frac{d y_{2}}{d t} & =\frac{a c p}{H}\left(1-u_{1}(t)\right) x_{2} y_{1}-v y_{2},
\end{aligned}
$$

where $1-u_{1}(t)$ describes the failure rate of prevention efforts. The per-capital recovery rate is $r u_{2}(t)$, where $0 \leqslant r \leqslant 1$ is the proportion of effective treatment.

\subsection{Optimal control problem}

Let

$$
J\left(u_{1}, u_{2}\right)=\int_{0}^{T}\left(A_{1} x_{2}(t)+B_{1} u_{1}^{2}(t)+B_{2} u_{2}^{2}(t)\right) d t
$$

We find an optimal control $\left(u_{1}^{*}, u_{2}^{*}\right)$ such that

$$
J\left(u_{1}^{*}, u_{2}^{*}\right)=\min \left\{J\left(u_{1}, u_{2}\right) \mid\left(u_{1}, u_{2}\right) \in \Gamma\right\},
$$


where the control set is

$$
\Gamma=\left\{\left(u_{1}, u_{2}\right) \mid u_{i}(t) \text { is a piece-wise continuous on }[0, T] \text { and } 0 \leqslant u_{i}(t) \leqslant 1, i=1,2\right\} \text {. }
$$

\subsection{Existence of optimal control}

Theorem 4.1. Consider the objective functional $\mathrm{J}$ with $\left(\mathrm{u}_{1}, \mathrm{u}_{2}\right) \in \Gamma$ subject to the constraint state system. Then, there exists $\left(u_{1}^{*}, u_{2}^{*}\right) \in \Gamma$ such that $J\left(u_{1}^{*}, u_{2}^{*}\right)=\min \left\{J\left(u_{1}, u_{2}\right),\left(u_{1}, u_{2}\right) \in \Gamma\right\}$.

Proof. Since the state variables and controls are continuous, by Peano's existence theorem the sets of controls and corresponding state variables are nonempty. Note that $\Gamma$ is convex and closed by the definition. Since we restrict the state variables in $\Omega$ and by the definition of $\Gamma, 0 \leqslant u_{i} \leqslant 1$, the state system with controls is bounded by a linear function. We easily see that the following inequality for the integrand of the cost functional

$$
A_{1} x_{2}+B_{1} u_{1}^{2}+B_{2} u_{2}^{2} \geqslant c_{1}\left(\left|u_{1}\right|^{2}+\left|u_{2}\right|^{2}\right)^{\beta / 2}-c_{1}
$$

for some $c_{1}>0, c_{2}>0$, and $\beta>1$. Also, let $L\left(x_{2}, u_{1}, u_{2}\right)=A_{1} x_{2}+B_{1} u_{1}^{2}+B_{2} u_{2}^{2}$. Then, we have the following inequality:

$$
\mathrm{L}\left(\mathrm{x}_{2}, \lambda \mathrm{u}_{1}+(1-\lambda) \mathrm{u}_{1}^{\prime}, \lambda \mathrm{u}_{2}+(1-\lambda) \mathrm{u}_{2}^{\prime}\right) \leqslant \lambda \mathrm{L}\left(\mathrm{x}_{2}, \mathrm{u}_{1}, \mathrm{u}_{2}\right)+(1-\lambda) \mathrm{L}\left(\mathrm{x}_{2}, \mathrm{u}_{1}^{\prime}, \mathrm{u}_{2}^{\prime}\right) .
$$

Thus, we conclude that the integrand is convex.

By theorem 4.1 in Fleming and Rishel [24], there exists $\left(u_{1}^{*}, u_{2}^{*}\right) \in \Gamma$ such that $J\left(u_{1}^{*}, u_{2}^{*}\right)=\min \left\{J\left(u_{1}, u_{2}\right)\right.$, where $\left.\left(u_{1}, u_{2}\right) \in \Gamma\right\}$.

\subsection{Optimality system}

Let $Z=(X, Y) \in \Omega$ where $X=\left(x_{1}, x_{2}, x_{3}\right)$ and $Y=\left(y_{1}, y_{2}\right), \Pi=\left(\lambda_{1}, \lambda_{2}, \lambda_{3}, \lambda_{4}, \lambda_{5}\right)$, and $U=\left(u_{1}, u_{2}\right) \in \Gamma$. We define a Hamiltonian as follows.

$$
\begin{aligned}
& L(Z, U, \Pi)=A_{1} x_{2}+B_{1} u_{1}^{2}+B_{2} u_{2}^{2}+\lambda_{1}\left[\delta_{1}-\frac{a b}{H}\left(1-u_{1}(t)\right) x_{1} y_{2}-\frac{\beta}{H} x_{1} x_{2}-\eta x_{1}\right] \\
& +\lambda_{2}\left[\theta\left(\frac{\mathrm{ab}}{\mathrm{H}}\left(1-\mathrm{u}_{1}(\mathrm{t})\right) \mathrm{x}_{1} \mathrm{y}_{2}+\frac{\beta}{\mathrm{H}} \mathrm{x}_{1} \mathrm{x}_{2}\right)-\gamma \mathrm{x}_{2}-\eta x_{2}-r \mathrm{u}_{2}(\mathrm{t}) \mathrm{x}_{2}\right] \\
& +\lambda_{3}\left[(1-\theta)\left(\frac{\mathrm{ab}}{\mathrm{H}}\left(1-\mathrm{u}_{1}(\mathrm{t})\right) \mathrm{x}_{1} \mathrm{y}_{2}+\frac{\beta}{\mathrm{H}} \mathrm{x}_{1} \mathrm{x}_{2}\right)+\gamma \mathrm{x}_{2}-\eta x_{3}+r \mathrm{u}_{2}(\mathrm{t}) \mathrm{x}_{2}\right] \\
& +\lambda_{4}\left[\delta_{2}-\frac{a c p}{H}\left(1-u_{1}(t)\right) x_{2} y_{1}-v y_{1}\right]+\lambda_{5}\left[\frac{a c p}{H}\left(1-u_{1}(t)\right) x_{2} y_{1}-v y_{2}\right] .
\end{aligned}
$$

Theorem 4.2. Given an optimal control pair $\left(\mathrm{u}_{1}^{*}, \mathrm{u}_{2}^{*}\right)$ and solutions $\mathrm{x}_{1}, \mathrm{x}_{2}, \mathrm{x}_{3}, \mathrm{y}_{1}$, and $\mathrm{y}_{2}$, there exist adjoint variables $\Pi$ satisfying

$$
\begin{aligned}
\dot{\lambda_{1}}= & \lambda_{1}\left(-\left(\frac{a b\left(1-u_{1}\right) x_{1} y_{2}}{H^{2}}-\frac{a b\left(1-u_{1}\right) y_{2}}{H}-\eta+\frac{\beta x_{1} x_{2}}{H^{2}}-\frac{\beta x_{2}}{H}\right)\right) \\
& -\theta \lambda_{2}\left(-\frac{a b\left(1-u_{1}\right) x_{1} y_{2}}{H^{2}}+\frac{a b\left(1-u_{1}\right) y_{2}}{H}-\frac{\beta x_{1} x_{2}}{H^{2}}+\frac{\beta x_{2}}{H}\right) \\
& -(1-\theta) \lambda_{3}\left(-\frac{a b\left(1-u_{1}\right) x_{1} y_{2}}{H^{2}}+\frac{a b\left(1-u_{1}\right) y_{2}}{H}-\frac{\beta x_{1} x_{2}}{H^{2}}+\frac{\beta x_{2}}{H}\right) \\
& -\frac{a c \lambda_{4} p\left(1-u_{1}\right) x_{2} y_{1}}{H^{2}}+\frac{a c \lambda_{5} p\left(1-u_{1}\right) x_{2} y_{1}}{H^{2}}, \\
\dot{\lambda_{2}}= & -\lambda_{2}\left(\theta\left(-\frac{a b\left(1-u_{1}\right) x_{1} y_{2}}{H^{2}}-\frac{\beta x_{1} x_{2}}{H^{2}}+\frac{\beta x_{1}}{H}\right)-\gamma-\eta-r u_{2}\right) \\
& -\lambda_{3}\left((1-\theta)\left(-\frac{a b\left(1-u_{1}\right) x_{1} y_{2}}{H^{2}}-\frac{\beta x_{1} x_{2}}{H^{2}}+\frac{\beta x_{1}}{H}\right)+\gamma+r u_{2}\right)
\end{aligned}
$$




$$
\begin{aligned}
& -\lambda_{1}\left(\frac{a b\left(1-u_{1}\right) x_{1} y_{2}}{H^{2}}+\frac{\beta x_{1} x_{2}}{H^{2}}-\frac{\beta x_{1}}{H}\right)-\lambda_{4}\left(\frac{a c p\left(1-u_{1}\right) x_{2} y_{1}}{H^{2}}-\frac{a c p\left(1-u_{1}\right) y_{1}}{H}\right) \\
& -\lambda_{5}\left(\frac{a c p\left(1-u_{1}\right) y_{1}}{H}-\frac{a c p\left(1-u_{1}\right) x_{2} y_{1}}{H^{2}}\right)-A_{1}, \\
\dot{\lambda_{3}}= & -\lambda_{3}\left((1-\theta)\left(-\frac{a b\left(1-u_{1}\right) x_{1} y_{2}}{H^{2}}-\frac{\beta x_{1} x_{2}}{H^{2}}\right)-\eta\right) \\
& -\theta \lambda_{2}\left(-\frac{a b\left(1-u_{1}\right) x_{1} y_{2}}{H^{2}}-\frac{\beta x_{1} x_{2}}{H^{2}}\right)+\lambda_{1}\left(-\left(\frac{a b\left(1-u_{1}\right) x_{1} y_{2}}{H^{2}}+\frac{\beta x_{1} x_{2}}{H^{2}}\right)\right) \\
& -\frac{a c \lambda_{4} p\left(1-u_{1}\right) x_{2} y_{1}}{H^{2}}+\frac{a c \lambda_{5} p\left(1-u_{1}\right) x_{2} y_{1}}{H^{2}}, \\
\dot{\lambda_{4}}= & -\lambda_{4}\left(-\frac{a c p\left(1-u_{1}\right) x_{2}}{H}-v\right)-\frac{a c \lambda_{5} p\left(1-u_{1}\right) x_{2}}{H}, \\
\dot{\lambda_{5}}= & -\frac{a b \theta \lambda_{2}\left(1-u_{1}\right) x_{1}}{H}-\frac{a b(1-\theta) \lambda_{3}\left(1-u_{1}\right) x_{1}}{H}+\frac{a b \lambda_{1}\left(1-u_{1}\right) x_{1}}{H}+\lambda_{5} v,
\end{aligned}
$$

with the terminal conditions,

$$
\lambda_{i}(T)=0 \text { for } i=1, \ldots, 5 .
$$

Furthermore, $\mathrm{u}_{1}^{*}$ and $\mathrm{u}_{2}^{*}$ are represented by

$$
\begin{aligned}
& u_{1}^{*}=\max \left\{0, \min \left\{1,-\frac{a\left(-b \theta \lambda_{2} x_{1} y_{2}+b \theta \lambda_{3} x_{1} y_{2}+b \lambda_{1} x_{1} y_{2}-b \lambda_{3} x_{1} y_{2}+c \lambda_{4} p x_{2} y_{1}-c \lambda_{5} p x_{2} y_{1}\right)}{2 B_{1} H}\right\}\right\}, \\
& u_{2}^{*}=\max \left\{0, \min \left\{1, \frac{r x_{2}\left(\lambda_{2}-\lambda_{3}\right)}{2 B_{2}}\right\}\right\} .
\end{aligned}
$$

Proof. By Pontryagin's Principle we get the adjoint system

$$
\dot{\lambda_{1}}=-\frac{\partial \mathrm{L}}{\partial x_{1}}, \dot{\lambda_{2}}=-\frac{\partial \mathrm{L}}{\partial x_{2}}, \dot{\lambda_{3}}=-\frac{\partial \mathrm{L}}{\partial x_{4}}, \dot{\lambda_{4}}=-\frac{\partial \mathrm{L}}{\partial \mathrm{y}_{1}}, \dot{\lambda_{5}}=-\frac{\partial \mathrm{L}}{\partial \mathrm{y}_{2}}
$$

with zero final time conditions. To get the formula of the optimal control, $\left(u_{1}^{*}, u_{2}^{*}\right)$, we solve the equations,

$$
\frac{\partial \mathrm{L}}{\partial \mathrm{u}_{1}}=0, \quad \frac{\partial \mathrm{L}}{\partial \mathrm{u}_{2}}=0,
$$

with the bounds of the controls given in (4.3).

\section{Numerical results}

For the numerical simulation, we use the values of parameters in Table 2. We choose weight constant values $A_{1}=1, B_{1}=50, B_{2}=50$ and $r=0.2$ in the nonlinear ordinary differential equations with the optimal controls (4.1) and the objective functional (4.2). The first set of values will result in $R_{0}<1$ and we will have a stable disease-free equilibrium, while the second set of values will result in $R_{0}>1$ and thus, we will have a stable endemic equilibrium point.

Figures 6 and 7 show the comparison between non-controlled and controlled cases when $R_{0}<1$. The number of infected human with the control is less than the number of non-controlled case in the whole time period. The number of infected human with the control goes to zero faster than the non-controlled case. Similarly, we see the same result for the mosquito population as shown in Figure 7.

Figures 8 and 9 show the comparison between non-controlled and controlled cases when $R_{0}>1$. The results are similar to the case when $R_{0}<1$. The significant result is that, we still have the endemic equilibrium points for the controlled case which are improved comparing to the non-control case. 
In this numerical example, we observe the expected result that the disease with optimal control can be controlled better than the non-controlled case. Especially, when $R_{0}>1$, the improvement is significant. In this paper, we considered two controls, $u_{1}$ for the prevention effort of the contact between human and mosquito and $u_{2}$ for the treatment for human.

Some authors have also considered other control mechanisms such as controlling the number of vectors of larval or adult, or controlling the contact between infected and healthy human $[10,26,56]$.

We search for two optimal control functions $u_{1}$ and $u_{2}$ for the prevention of the contact between human and vector and the treatment for the human population, respectively. For the case when $R_{0}<1$, we have the optimal control values, $\mathfrak{u}_{1}=0.0349$, and $\mathfrak{u}_{2}=0.8085$. For $R_{0}>1$ case, we have the optimal control values, $u_{1}=0.0277$ and $u_{2}=0.4813$. It mean that the prevention effort has lower efficacy in both cases when $R_{0}<1$ and $R_{0}>1$. Therefore, to optimize the number of infected and treated hosts, the treatment rate has to be increased.

We also search for optimal control function for the prevention, $\mathfrak{u}_{1}$, by setting $\mathfrak{u}_{2}=0$, that is, when there is no treatment. In this case if $\mathcal{R}_{0}<1$ then $u_{1}=0.1001$ and if $\mathcal{R}_{0}>1$ then $u_{1}=0.0387$. Moreover, we investigated the situation when the prevention effort of the contact between the human and mosquito $\left(u_{1}\right)$ is zero and the treatment $\left(u_{2}\right)$ is non-zero. As a result if $\mathcal{R}_{0}<1$ then $u_{2}=0.8085$ and if $\mathcal{R}_{0}>1$ then $\mathfrak{u}_{2}=0.4810$. Thus we can conclude that the treatment is more effective than the prevention when $\mathrm{R}_{0}<1$. Similarly, if $\mathcal{R}_{0}>1$ then preventing the infection by reducing the contact between the human and mosquito is better than providing treatment. However in order to get a much better result, both prevention and treatment controls should be considered.
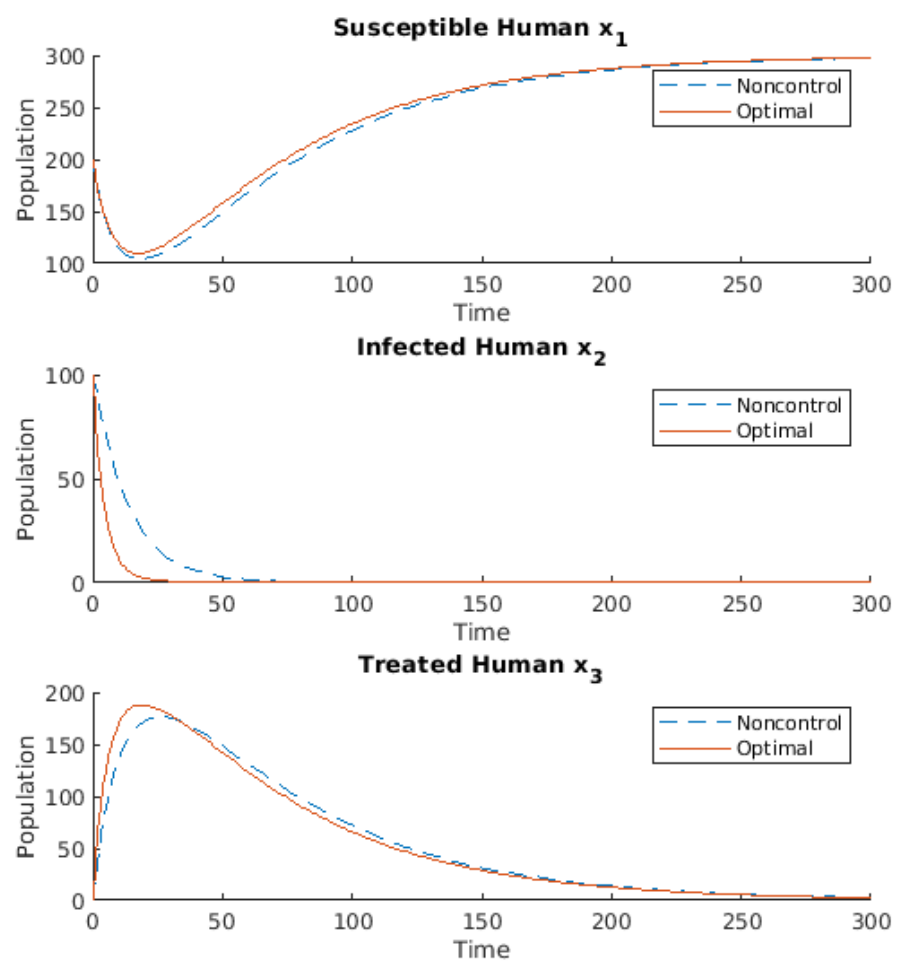

Figure 6: Comparison between with-control and non-control for human when $\mathrm{R}_{0}<1$. 

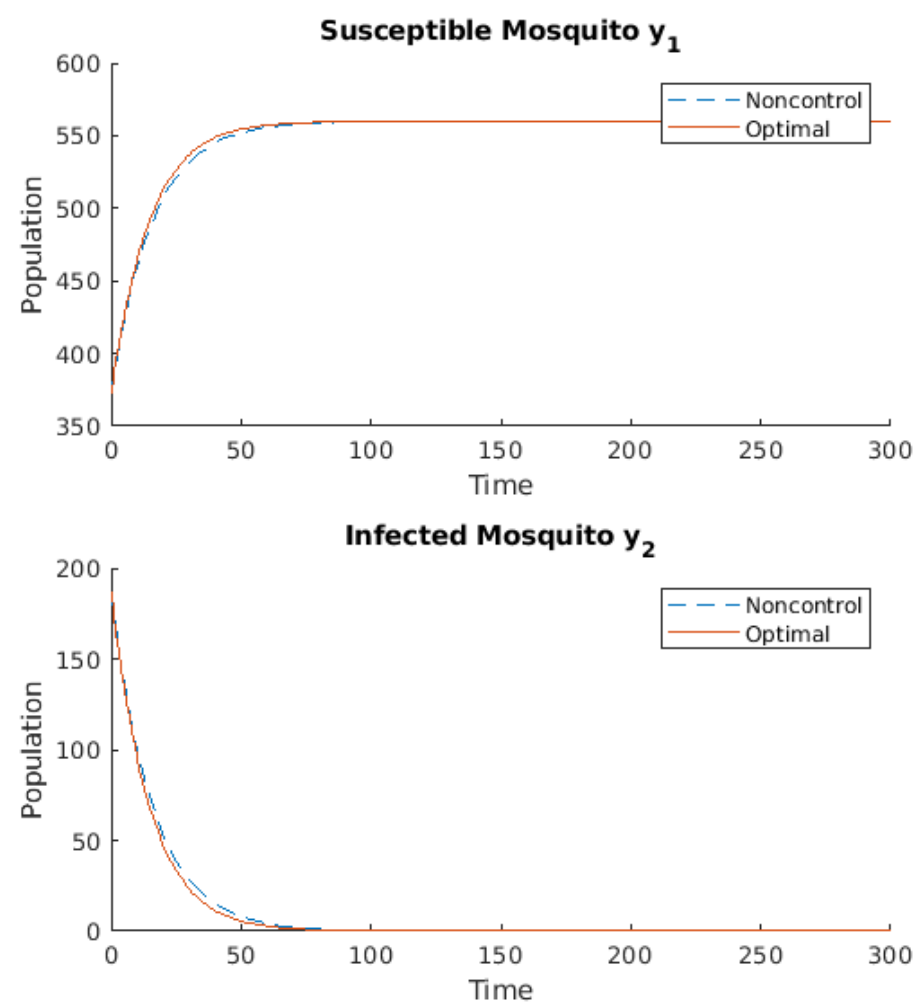

Figure 7: Comparison between with-control and non-control for mosquito when $\mathrm{R}_{0}<1$.
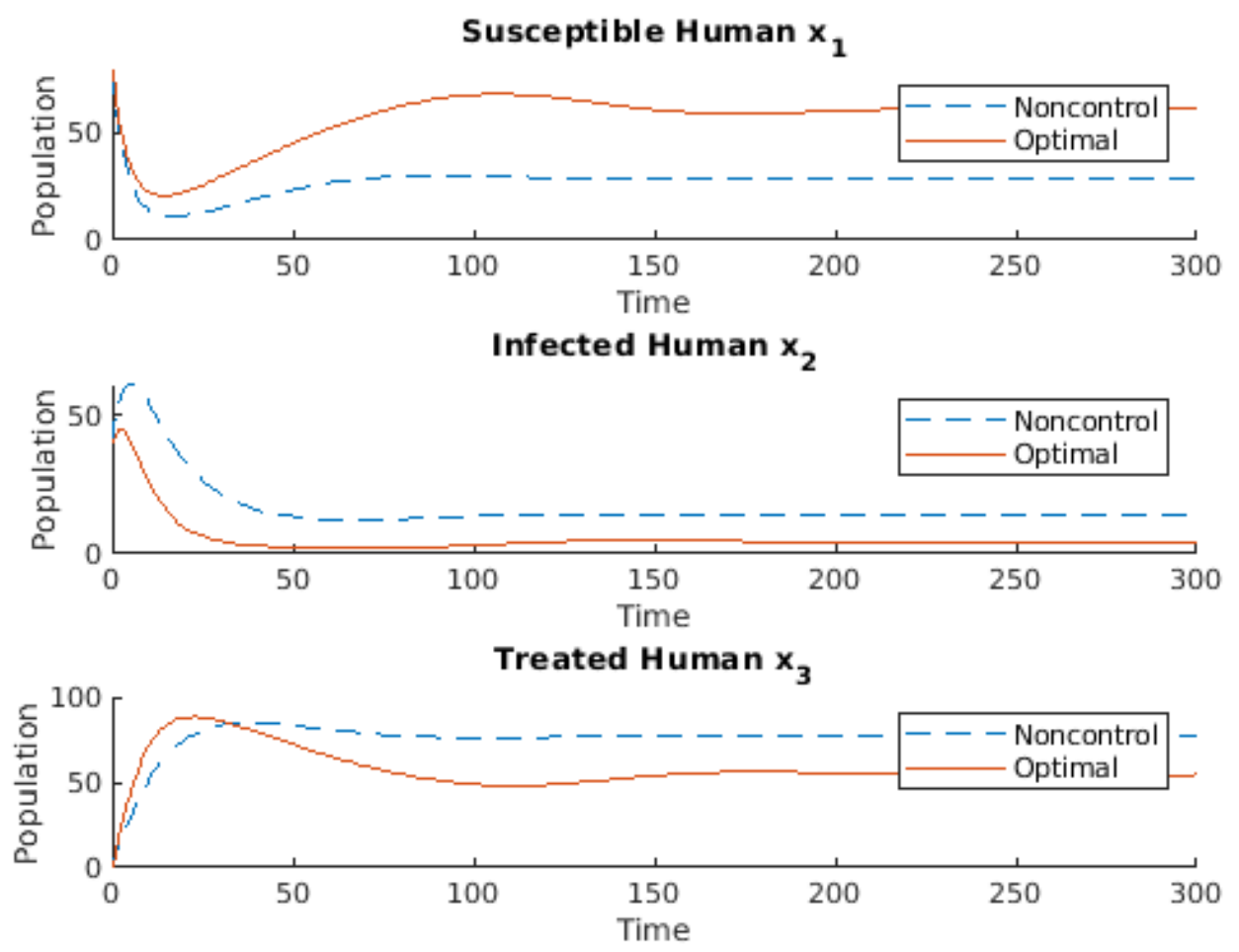

Figure 8: Comparison between with-control and non-control for human when $R_{0}>1$. 

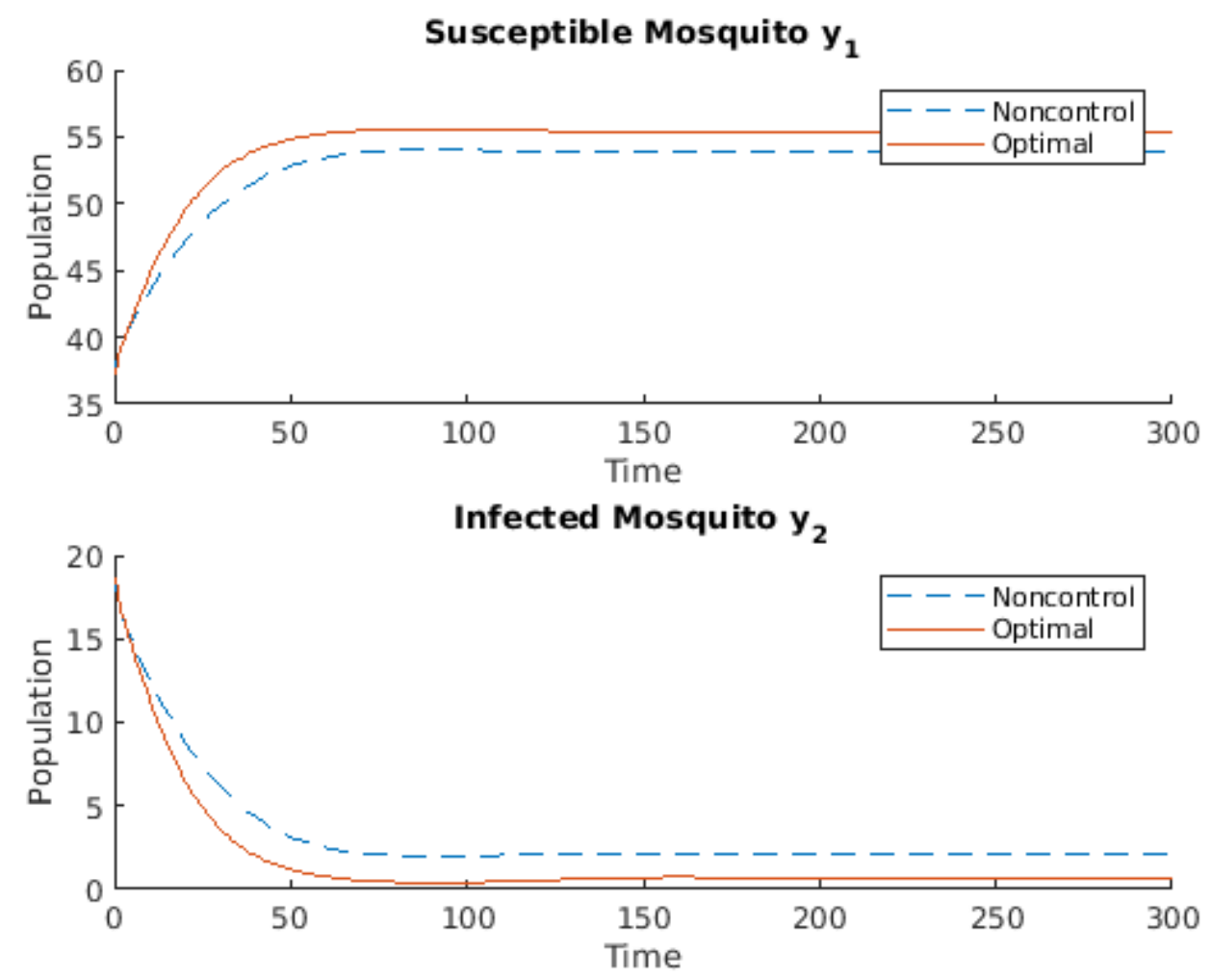

Figure 9: Comparison between with-control and non-control for mosquito when $R_{0}>1$.

\section{Discussion and Conclusion}

In this paper, a deterministic Zika virus model is proposed and analyzed. In particular, we calculated some of the most important epidemiological constants. This includes, the disease-free equilibrium point $\mathrm{E}_{0}$ which is the case when the pathogen has suffered extinction and in the long run, everyone in the population is susceptible and the epidemic equilibrium point $E_{1}$, in which the disease cannot be totally eradicated and remains in the population. Another important quantity that is derived and discussed in this paper is the basic reproductive number $\mathcal{R}_{0}$, which describes the average number of secondary infections caused by a single infectious individual during their entire infectious lifetime. It is also shown that both the local and global dynamics of the solution of the Zika model is completely determined by the value of $\mathcal{R}_{0}$. That is, if $\mathcal{R}_{0}<1$, then the disease-free equilibrium point is both locally and globally asymptotically stable, and thus there will be no infection eventually. On the other hand, if $\mathcal{R}_{0}>1$, the epidemic equilibrium point is also both locally and globally asymptotically stable and thus there will always be infection in the vector and host population. Generally, global stability of the epidemic equilibrium point is proved by constructing a suitable Lyapunov function $V(t)$ and claiming that $\frac{d V}{d t} \leqslant 0$. However, constructing an appropriate Lyapunov function is not always easy. Thus, in this paper we have used an alternative technique, the Poincare-Bendixon method, to show that the endemic equilibrium point is globally stable.

Since the two equilibrium points and the basic reproductive number depend on different parameters used in the model, it is important to know which parameter affects these quantities the most. In this regard, the sensitivity analysis of the equilibrium points and the basic reproductive number is discussed, and the result is supported by numerical examples.

We also studied optimal control of the deterministic Zika virus model. We considered two controls $u_{1}$ for the prevention effort of the contact between human and vector and $u_{2}$ for the treatment for human. We showed the existence of the optimal controls by using the result from [24]. To find $u_{1}$ and $u_{2}$, we 
established the optimality system by defining a Lagrangian (4.4). The optimality system is consisted of the initial conditions of $x_{1}, x_{2}, x_{3}, y_{1}, y_{2}$, and the adjoint system (4.5) with the terminal conditions (4.7) and the optimal conditions, $u_{1}$ and $u_{2}(4.8)$.

We compared the number of human and vectors with controls and without controls in Figures 6 and 7 when $R_{0}<1$ and in Figures 8 and 9 when $R_{0}>1$. From the figures, we can observe that the model with controls have better result than the model without controls. In the comparison, we have that the value of $u_{1}$ is 0.0349 and $u_{2}$ is 0.8085 . It means the treatment is more efficacy than the prevention.

Also, we discussed the case when we have a single control by setting $\mathfrak{u}_{1}=0$ and search for $\mathfrak{u}_{2}$. Similarly, we search for $u_{1}$ by setting $u_{2}=0$. In both cases we found the same result that the treatment is more efficient than the prevention.

In most infections caused by vectors, experimenting to come up with an excellent control measure is often difficult and time consuming. Thus, developing mathematical models that can help us to predict the spread of the infection is very important. These models also have significant role in informing policy and management decisions about the infection. However, as explained in [25, 33], even though there are a variety of mathematical tools for designing optimal strategies, it is difficult to put the results from mathematically motivated simplifications into practice. Additional research and follow up will be needed subsequently to understand the disease more fully and assess and update the response policy as needed. More detailed information on how to use the results of the optimal control in policy decision making can be found in $[25,33]$.

\section{References}

[1] B. M. Adams, H. T. Banks, M. Davidian, H.-D. Kwon, H. T. Tran, S. N. Wynne, E. S. Rosenberg, HIV dynamics: modeling, data analysis, and optimal treatment protocols, J. Comput. Appl. Math., 184 (2005), 10-49. 1

[2] R. M. Anderson, R. M. May, Infectious diseases of humans: dynamics and control, Oxford university press, (1992). 3.1

[3] N. T. J. Bailey, The mathematical theory of infectious diseases and its applications, Hafner Press, New York, (1975).

[4] S. Bewick, W. F. Fagan, J. Calabrese, F. Agusto, Zika virus: endemic versus epidemic dynamics and implications for disease spread in the Americas, BioRxiv, (2016). 1

[5] B. Blitvich, Arboviruses: Molecular Biology, Evolution and Control. Nikos Vasilakis and Duane J. Gubler, Am. J. Trop. Med. Hyg., 95 (2016), 488-489. 1

[6] I. I. Bogoch, O. J. Brady, M. U. G. Kraemer, M. German, M. I. Creatore, M. A. Kulkarni, J. S. Brownstein, S. R. Mekaru, S. I. Hay, E. Groot, A. Watts, K. Khan, Anticipating the international spread of Zika virus from Brazil, The Lancet, 387 (2016), 335-336. 1

[7] E. Bonyah, K. O. Okosun, Mathematical modeling of Zika virus, Asian Pac. J. Trop. Dis., 6 (2016), 673-679. 2

[8] S. E. B. Boret, R. Escalante, M. Villasana, Mathematical modelling of zika virus in Brazil, arXiv preprint arXiv:1708.01280, (2017), 26 pages. 2

[9] P. Brasil, J. P. Pereira, Jr., M. E. Moreira, R. M. Ribeiro Nogueira, L. Damasceno, M. Wakimoto, R. S. Rabello, S. G. Valderramos, U.-A. Halai, T. S. Salles, A. A. Zin, D. Horovitz, P. Daltro, M. Boechat, C. Raja Gabaglia, P. Carvalho de Sequeira, J.H. Pilotto, R. Medialdea-Carrera, D. Cotrim da Cunha, L. M. Abreu de Carvalho, M. Pone, A. Machado Siqueira, G.A. Calvet, A.E. Rodrigues Baião, E.S. Neves, P.R. Nassar de Carvalho, R. H. Hasue, P. B. Marschik, C. Einspieler, C. Janzen, J. D. Cherry, A. M. Bispo de Filippis, K. Nielsen-Saines, Zika virus infection in pregnant women in Rio de Janeiro, N. Engl. J. Med., 375 (2016), 2321-2334. 1

[10] E. H. Bussell, C. E. Dangerfield, C. A. Gilligan, N. J. Cunniffe, Applying optimal control theory to complex epidemiological models to inform real-world disease management, Philosophical Transactions of the Royal Society B, 374 (2019), 7 pages. 5

[11] G. Butler, P. Waltman, Persistence in dynamical systems, J. Differential Equations, 63 (1986), 255-263. 3.2, 3.2

[12] V.-M. Cao-Lormeau, A. Blake, S. Mons, S. Lastère, C. Roche, J. Vanhomwegen, T. Dub, Laure Baudouin, A. Teissier, P. Larre, A.-L. Vial, C. Decam, V. Choumet, S. K. Halstead, H. J. Willison, L. Musset, J.-C. Manuguerra, P. Despres, E. Fournier, H.-P. Mallet, D. Musso, A. Fontanet, J. Neil, F. Ghawché, Guillain-Barré Syndrome outbreak associated with Zika virus infection in French Polynesia: a case-control study, The Lancet, 387 (2016), 1531-1539. 1

[13] S. Cauchemez, M. Besnard, P. Bompard, T. Dub, P. Guillemette-Artur, D. Eyrolle-Guignot, H. Salje, M. D. V. Kerkhove, V. Abadie, C. Garel, A. Fontanet, H.-P. Mallet, Association between Zika virus and microcephaly in French Polynesia, 2013-15: a retrospective study, The Lancet, 387 (2016), 2125-2132. 1

[14] L. Cesari, Asymptotic behavior and stability problems in ordinary differential equations, Springer Science \& Business Media, (2012). 3.2

[15] B. Chachuat, Nonlinear and dynamic optimization: From theory to practice, Laboratoire d'Automatique, École Polytechnique Fédérale de Lausanne, (2007). 1 
[16] R. V. Culshaw, S. Ruan, R. J. Spiteri, Optimal HIV treatment by maximising immune response, J. Math. Biol., 48 (2004), 545-562. 1

[17] E. X. DeJesus, C. Kaufman, Routh-Hurwitz criterion in the examination of eigenvalues of a system of nonlinear ordinary differential equations, Phys. Rev. A, 35 (1987), 5288-5290. 3.2

[18] G. W. A. Dick, S. F. Kitchen, A. J. Haddow, Zika virus (I). Isolations and serological specificity, Trans. R. Soc. Trop. Med. Hyg., 46 (1952), 509-520.

[19] O. Diekmann, J. A. P. Heesterbeek, Mathematical epidemiology of infectious diseases: model building, analysis and interpretation, John Wiley \& Sons, (2000). 3.1

[20] O. Diekmann, J. A. P. Heesterbeek, J. A. J. Metz, On the definition and the computation of the basic reproduction ratio $R$ 0 in models for infectious diseases in heterogeneous populations, J. Math. Biol., 28 (1990), 365-382. 3.1

[21] M. R. Duffy, T.-H. Chen, W. Thane Hancock, A. M. Powers, J. L. Kool, R. S. Lanciotti, M. Pretrick, M. Marfel, S. Holzbauer, C. Dubray, L. Guillaumot, A. Griggs, M. Bel, A. J. Lambert J. Laven, O. Kosoy, M.S., A. Panella, B. J. Biggerstaff, M. Fischer, E. B. Hayes, Zika virus outbreak on Yap Island, federated states of Micronesia, N. Engl. J. Med., 360 (2009), 2536-2543. 1

[22] H. Elsaka, E. Ahmed, A fractional order network model for ZIKA, BioRxiv, (2016), 10 pages. 1

[23] A. S. Fauci, D. M. Morens, Zika virus in the Americas-yet another arbovirus threat, N. Engl. J. Med., 374 (2016), 601-604.

[24] W. H. Fleming, R. W. Rishel, Deterministic and stochastic optimal control, Springer Science \& Business Media, (2012). $4.3,6$

[25] H. Gaff, E. Schaefer, Optimal control applied to vaccination and treatment strategies for various epidemiological models, Math. Biosci. Eng., 6 (2009), 469-492. 6

[26] S. R .Gani, S. V. Halawar, Optimal Control Analysis of Deterministic and Stochastic SIS Epidemic Model with Vaccination, Int. J. Stats. Med. Res., 12 (2017), 251-263. 5

[27] D. Gao, Y. Lou, D. He, T. C. Porco, Y. Kuang, G. Chowell, S. Ruan, Prevention and control of Zika as a mosquito-borne and sexually transmitted disease: a mathematical modeling analysis, Sci. Rep., 6 (2016), 10 pages. 1, 1

[28] D. J. Gubler, The changing epidemiology of yellow fever and dengue, 1900 to 2003: full circle, Comp. Immunol. Microbiol. Infect. Dis., 27 (2004), 319-330. 1

[29] A. D. Haddow, A. J. Schuh, C. Y. Yasuda, M. R. Kasper, V. Heang, R. Huy , H. Guzman, R. B. Tesh, S. C. Weaver, Genetic characterization of Zika virus strains: geographic expansion of the Asian lineage, PLOS Negl. Trop. Dis., 6 (2012), 7 pages. 1

[30] J. Heukelbach, C. H. Alencar, A. A. Kelvin, W. K. de Oliveira, L. P. de Góes Cavalcanti, Zika virus outbreak in Brazil, J. Infect. Dev. Ctries, 10 (2016), 116-120. 1

[31] H. R. Joshi, Optimal control of an HIV immunology model, Optim. Control Appl. Methods, 23 (2002), 199-213. 1

[32] E. Jung, S. Lenhart, Z. Feng, Optimal control of treatments in a two-strain tuberculosis model, Discrete Contin. Dyn. Syst. Ser. B, 2 (2002), 473-482. 1

[33] S. M. Kassa, A. Ouhinou, The impact of self-protective measures in the optimal interventions for controlling infectious diseases of human population, J. Math. Biol., 70 (2015), 213-236. 6

[34] C. L. Keighley, R. B. Saunderson, J. Kok, D. E. Dwyer, Viral exanthems, Curr. Opin. Infect. Dis., 28 (2015), $139-150$. 1

[35] I. E. Kibona, C.Yang, SIR model of spread of Zika virus infections: ZIKV linked to microcephaly simulations, Health, 9 (2017), 1190-1210. 2

[36] D. Kirschner, S. Lenhart, S. Serbin, Optimal control of the chemotherapy of HIV, J. Math. Biol., 35 (1997), 775-792. 1

[37] A. J. Kucharski, S. Funk, R. M. Eggo, H.-P. Mallet, W. J. Edmunds, E. J. Nilles, Transmission dynamics of Zika virus in island populations: a modelling analysis of the 2013-14 French Polynesia outbreak, PLOS Negl. Trop. Dis., 10 (2016), 15 pages. 1

[38] J. P. LaSalle, The stability of dynamical systems, Society for Industrial and Applied Mathematics, Philadelphia,Pa.., (1976). 3.2

[39] S. Lenhart, J. T. Workman, Optimal control applied to biological models, Chapman \& Hall/CRC, Boca Raton, FL, (2007). 1

[40] M. Y. Li, L. Wang, Global stability in some SEIR epidemic models, Springer, New York, (2002), 295-311. 3.2, 3.2, 3.2, 3.2

[41] D. R. Lucey, L. O. Gostin, The emerging Zika pandemic: enhancing preparedness, Jama, 315 (2016), 865-866. 1

[42] J. C. Miller, Mathematical models of SIR disease spread with combined non-sexual and sexual transmission routes, Infect. Dis. Model., 2 (2017), 35-55. 2

[43] J. Mlakar, M. Korva, N. Tul, M. Popović, M. Poljšak-Prijatelj, J. Mraz, M. Kolenc, K. Resman Rus, T. Vesnaver Vipotnik, V. Fabjan Vodušek, A. Vizjak, J. Pižem, M. Petrovec, T. Avšič Županc, Zika virus associated with microcephaly, N. Engl. J. Med., 374 (2016), 951-958.

[44] V. M. Moreno, B. Espinoza, D. Bichara, S. A. Holechek, C. Castillo-Chavez, Role of short-term dispersal on the dynamics of Zika virus in an extreme idealized environment, Infect. Dis. Model., 2 (2017), 21-34. 1

[45] D. Musso, D. J. Gubler, Zika virus, Clin. Microbiol. Rev., 29 (2016), 487-524. 1

[46] E. Oehler, E. Fournier, I. Leparc-Goffart, P. Larre, S. Cubizolle, C. Sookhareea, S. Lastère, F. Ghawche, Increase in cases of Guillain-Barré syndrome during a Chikungunya outbreak, French Polynesia, 2014 to 2015, Eurosurveillance, 20 
(2015), 2 pages 1

[47] E. Oehler, L. Watrin, P. Larre, I. Leparc-Goffart, S. Lastère, F. Valour, L. Baudouin, H. P. Mallet, D. Musso, F. Ghawche, Zika virus infection complicated by Guillain-Barre syndrome-case report, French Polynesia, December 2013, Eurosurveillance, (2014), 3 pages. 1

[48] M. Ozair, A. A. Lashari, I. H. Jung, K. O. Okosun Stability analysis and optimal control of a vector-borne disease with nonlinear incidence, Discrete Dyn. Nat. Soc., 2012 (2012), 21 pages. 1

[49] T. A. Perkins, A. S. Siraj, C. W. Ruktanonchai, M. U. G. Kraemer, A. J. Tatem, Model-based projections of Zika virus infections in childbearing women in the Americas, Nat. Microbiol., 1 (2016), 7 pages. 1

[50] P. Reiter, 25 Surveillance and Control of Urban Dengue Vectors, Dengue and Dengue Hemorrhagic Fever, (2014). 1

[51] S. A. Ritchie, 24 Dengue Vector Bionomics: Why Aedes aegypti is Such a Good Vector, Dengue and Dengue Hemorrhagic Fever, (2014). 1

[52] H. S. Rodrigues, Optimal control and numerical optimization applied to epidemiological models, arXiv preprint arXiv:1401.7390, (2014). 1

[53] D. P. Shutt, C. A. Manore, S. Pankavich, A. T. Porter, S. Y. Del Valle, Estimating the reproductive number, total outbreak size, and reporting rates for Zika epidemics in South and Central America, Epidemics, 21 (2017), 63-79. 1, 1

[54] H. L. Smith, H. R. Thieme, Dynamical systems and population persistence, American Mathematical Soc., (2011). 3.2

[55] P. Suparit, A. Wiratsudakul, C. Modchang, A mathematical model for Zika virus transmission dynamics with a timedependent mosquito biting rate, Theor. Biol. Med. Model., 15 (2018), 11 pages. 1

[56] L. S. Tusting, J. Thwing, D. Sinclair, U. Fillinger, J. Gimnig, K. E. Bonner, C. Bottomley, S. W. Lindsay, Mosquito larval source management for controlling malaria, Cochrane Database Syst. Rev., (2013). 5

[57] P. Van den Driessche, J. Watmough, Reproduction numbers and sub-threshold endemic equilibria for compartmental models of disease transmission, Math. Biosci., 180 (2002), 29-48. 3.2

[58] D. A. M. Villela, L. S. Bastos, L. M. De Carvalho, O. G. Cruz, M. F. C. Gomes, B. Durovni, M. C. Lemos, V. Saraceni, F. C. Coelho, C. T. Codeco, Zika in Rio de Janeiro: Assessment of basic reproduction number and comparison with dengue outbreaks, Epidemiol. Infect., 145 (2017), 1649-1657. 1

[59] P. Waltman, A brief survey of persistence in dynamical systems, Springer, Berlin, (1991). 3.2

[60] A. Wiratsudakul, P. Suparit, C. Modchang, Dynamics of Zika virus outbreaks: an overview of mathematical modeling approaches, PeerJ, 6 (2018), 30 pages. 1, 2

[61] J. Zabczyk, Mathematical control theory: an introduction, Springer Science \& Business Media, (2009). 1 